\title{
PREPRINT
}

Author-formatted, not peer-reviewed document posted on 26/04/2021

DOI: https://doi.org/10.3897/arphapreprints.e67869

\section{Distribution and identification of the}

\section{species in the genus Helicops Wagler,}

1830 (Serpentes, Colubridae,

\author{
Xenodontinae)
}

\section{Yannis Schöneberg, Gunther Köhler}

\section{Disclaimer on biological nomenclature and use of preprints}

The preprints are preliminary versions of works accessible electronically in advance of publication of the final version. They are not issued for purposes of botanical, mycological or zoological nomenclature and are not effectively/validly published in the meaning of the Codes. Therefore, nomenclatural novelties (new names) or other nomenclatural acts (designations of type, choices of priority between names, choices between orthographic variants, or choices of gender of names) should NOT be posted in preprints. The following provisions in the Codes of Nomenclature define their status:

International Code of Nomenclature for algae, fungi, and plants (ICNafp)

Article 30.2: "An electronic publication is not effectively published if there is evidence within or associated with the publication that its content is merely preliminary and was, or is to be, replaced by content that the publisher considers final, in which case only the version with that final content is effectively published." In order to be validly published, a nomenclatural novelty must be effectively published (Art. 32.1(a)); in order to take effect, other nomenclatural acts must be effectively published (Art. 7.10, 11.5, 53.5, 61.3, and 62.3).

International Code of Zoological Nomenclature (ICZN)

Article: 21.8.3: "Some works are accessible online in preliminary versions before the publication date of the final version. Such advance electronic access does not advance the date of publication of a work, as preliminary versions are not published (Article 9.9)". 


\title{
Distribution and identification of the species in the genus Helicops Wagler, 1830 (Serpentes, Colubridae, Xenodontinae)
}

\author{
Yannis Schöneberg ${ }^{\ddagger} \S$, Gunther Köhler§ \\ ‡ Johann Wolfgang Goethe-University, Frankfurt am Main, Germany \\ § Senckenberg Society for Nature Research, Frankfurt am Main, Germany \\ Corresponding author: Yannis Schöneberg (yannis.schoeneberg@gmx.de)
}

\section{Abstract \\ Background}

The aquatic snakes of the genus Helicops are widely distributed throughout northern South America but understudied concerning many aspects, including morphological traits and distribution. The most recent publication that provided an identification key to all species of Helicops is more than 50 years old. The key is of limited value today since it includes taxa no longer recognized and lacks 8 of the 19 species currently recognized. There never was a publication trying to summarize the distribution information of all species of Helicops. Most of the knowledge on these species is distributed over many small publications, like observation notes.

\section{New information}

Here we present point distribution maps, an identification key and comments on identification for all species in this genus based on the results of a comprehensive literature review of over 300 scientific publications and own examinations. Our examinations comprise 190 specimens of 10 of the 19 currently recognized species and one Helicops sp. Furthermore, we report range extensions for the species $H$. angulatus, $H$. danieli, $H$. infrataeniatus, $H$. leopardinus, $H$. pastazae and $H$. polylepis.

\section{Keywords}

annotated list, aquatic snakes, distribution maps, identification key, morphology, neotropics, pholidosis, taxonomy 


\section{Introduction}

The water snakes of the genus Helicops are widely distributed in the northern half of South America. The genus consists of 19 species of aquatic snakes inhabiting nearly all kinds of waterbodies in their distribution range. They seem to be rather generalist, inhabiting various bodies of water, from small ponds and puddles to slow flowing streams also in urban areas (Koski et al. 2016, Hernández-Ruiz et al. 2014, França et al. 2012). However, for many species not much more than the species description is known. The genus Helicops forms the Hydropsini together with the genera Hydrops and Pseudoeryx. The Hydropsini are part of the subfamily Dipsadinae, and characterized by the wide origin of the adductor mandibulae externus superficialis (Di Pietro et al. 2014). The genus Helicops consists of 19 species which all share the combination of having the eyes and nostrils directed dorsally on the top of the head, a single internasal scale, a divided cloacal shield, and at least some dorsal scales keeled (Costa et al. 2016). The first description of the species in this genus was Helicops angulatus, which was described as Coluber angulatus and $C$. alidras by Linnaeus (1758). In 1830, C. angulatus, and other species of the genera Coluber and Natrix were assigned to the newly created genus Helicops by Wagler (1830). At the time the last identification key was published by Peters and Orejas-Miranda (1970), 13 species were recognized, including the no longer recognized $H$. hogei and $H$. pictiventris (Rossman 2002). Since then, $H$. infrataeniatus has been raised to species level again and seven new species have been described $(H$. apiaka, $H$. boitata, $H$. nentur, $H$. phantasma, $H$. petersi, $H$. tapajonicus, $H$. yacu), more than half of them in the past 10 years $(H$. apiaka, $H$. boitata, $H$. nentur, $H$. phantasma). For all these seven species not much more than the species description is known. This and the amount of recently described new species shows the incompleteness of the taxonomic knowledge for this genus. Most of the knowledge is scattered across numerous publications. Especially distribution information is mostly contained in observation notes. Therefore, we want to present a basis for further taxonomic studies, by providing point distribution maps, an annotated checklist, and an identification key based on a comprehensive literature review and own observations.

\section{Materials and methods}

The assessment of the status of the species in the genus Helicops is based on the morphological examination of 190 specimens of 10 of the 19 currently recognized species in this genus as well as on a comprehensive literature review. The specimens are located in six herpetological museum collections in Germany: Senckenberg Research Institution Frankfurt (SMF); Senckenberg Naturhistorische Sammlungen Dresden (MTKD); Zoologisches Forschungsmuseum Alexander König in Bonn (ZFMK); Zoologische Staatssammlung München (ZSM); Staatliches Museum für Naturkunde Stuttgart (SMNS); Naturkundemuseum Berlin (NMB). 
The examined morphologic characters were: snout-vent length (SVL), tail length (TL), the ratio between tail length and snout-vent length (TL/SVL), number of ventral shields (VE), number of subcaudal scales (SC), presence of subcaudal keels (SCK); number of preoculars (PRO), number of postoculars (PSO), number of loreals (LO), number of anterior temporals (AT), number of posterior temporals (PT), number of supralabials (SL), number of supralabials in contact with the eye (SL+E), number of infralabials (IL), number of dorsal scale rows at midbody (DSM), presence of dorsal keels at midbody (DKM), number of dorsal scale rows approximately a head length prior to cloaca (DSP), presence of dorsal keels approximately a head length prior to cloaca (DKP), if cloacal plate is divided $(C L)$, if nasal scale is divided, semidivided or entire (NA), presence of intergenials (IG). The number of ventral scales, number of subcaudal scales, snout-vent length, tail length and the ratio between snout vent length and tail length were recorded separately for male and female specimens. Measurements were taken using a tape measure with the accuracy of $0.1 \mathrm{~cm}$. Ventrals were counted as proposed by Dowling (1951). The sex of the specimens was determined by exterior examination of the shape of the base of tail (tail bulge caused by presence of hemipenes in males, such bulge absent in females). The results of our examinations are available in Suppl. material 1. For specimens with a damaged tail, no tail measurements were done. The head scutellation was recorded for each side seperately.

The presented information on the species distribution is based on the locality data of the specimens examined and on literature data. One species locality was found browsing iNaturalist, the identification was made on the unique coloration of that species. Only records were included for which a reliable description of the locality or coordinates were available. All information on the literature references to the respective distribution point is listed in Suppl. material 2.

The distribution maps were created using QGIS 3.12.2 (Bucureşti) and the maps freely available at naturalearthdata.com.

The identification key was created using the morphological data gathered by specimens of Helicops examined by Y.S. and literature data. The literature references used for morphology are listed in Suppl. material 3.

\section{Data resources}

The Suppl. materials 2, 1 are tables in a tab delimited text format. Suppl. material 1contains the examination results for each specimen. It has columns with following headers: Species; Catalog numb.; Sex; Snout-venth length [mm]; Tail length [mm]; TL/SVL; Ventrals; Subcaudals; Subcaudal keels; Preoculars right; Preoculars left; Loreals right; Loreals left; Postoculars right; Postoculars left; Anterior Temporals right; Anterior Temporals left; Posterior Temporals right; Posterior Temporals left; Supralabials right; Supralabials left ; Supralabials + Eye right; Supralabials + Eye left; Sublabials right; Sublabials left; Dorsal scale rows at midbody; Dorsal keels at midbody; Dorsal scale rows at posterior body; Dorsal keels at posterior body; Analplate; Intergenials. The content of the columns follows the description of the examined morphological characters in the Methods section.Suppl. 
material 2 contains all distribution points extracted from literature und their reference. It has columns with following headers: Species; Country; Province; Locality; Locality notes; Latitude (DD); Longitude (DD); Latitude (DMS); Longitude (DMS); Literature; Online version. Most of the columns are selfexplanatory. The columns Latitude (DD) and Longitude (DD) contain the coordinates in Decimal degree and the columns Latitude (DMS) and Longitude (DMS) contain the coordinates in Degrees Minutes Seconds format.

Suppl. material 3 is a plain text file containing all the references used for the morphological assessment. It contains each reference in a seperate line.

\section{Taxon treatments}

\section{Helicops angulatus (Linnaeus, 1758)}

\section{Materials}

a. $\quad$ scientificName: Helicops angulatus (Linnaeus, 1758); country: Brazil; individualCount: 1; sex: male; catalogNumber: MTKD 15294; recordedBy: Fritzsche leg.; institutionID: MTKD

b. $\quad$ scientificName: Helicops angulatus (Linnaeus, 1758); country: Brazil; stateProvince: unknown province; locality: Ega ad Amazonas; year: 1831; individualCount: 1; sex: female; catalogNumber: MTKD 15509; recordedBy: Poeppig leg.; institutionID: MTKD

c. ScientificName: Helicops angulatus (Linnaeus, 1758); country: Peru; individualCount: 1; sex: female; catalogNumber: MTKD 41670; institutionID: MTKD

d. ScientificName: Helicops angulatus (Linnaeus, 1758); country: Bolivia; stateProvince: Santa Cruz; locality: Nuflo de Chavez, RPPN San Sebastian; verbatimLocality: 524; verbatimLatitude: $-16^{\circ} 23.263$; verbatimLongitude: $-61^{\circ} 59.983$; year: 2006 ; individualCount: 1; sex: female; catalogNumber: SMF 100016; recordedBy: M. Jansen leg.; institutionID: SMF

e. $\quad$ scientificName: Helicops angulatus (Linnaeus, 1758); individualCount: 1; sex: female; catalogNumber: SMF 17817; institutionID: SMF

f. $\quad$ scientificName: Helicops angulatus (Linnaeus, 1758); individualCount: 1; sex: female; catalogNumber: SMF 17818; institutionID: SMF

g. scientificName: Helicops angulatus (Linnaeus, 1758); individualCount: 1; sex: female; catalogNumber: SMF 17819; institutionID: SMF

h. $\quad$ scientificName: Helicops angulatus (Linnaeus, 1758); individualCount: 1; sex: male; catalogNumber: SMF 17820; institutionID: SMF

i. $\quad$ scientificName: Helicops angulatus (Linnaeus, 1758); individualCount: 1; sex: female; catalogNumber: SMF 32409; institutionID: SMF

j. $\quad$ scientificName: Helicops angulatus (Linnaeus, 1758); individualCount: 1; sex: female; catalog Number: SMF 32410; institutionID: SMF

k. ScientificName: Helicops angulatus (Linnaeus, 1758); individualCount: 1; sex: female; catalogNumber: SMF 40029; institutionID: SMF

I. scientificName: Helicops angulatus (Linnaeus, 1758); country: Peru; stateProvince: Ucayali; locality: Bolognesi (Campamento); verbatimLocality: 230; verbatimLatitude: $-10^{\circ} 6.217$; verbatimLongitude: $-73^{\circ} 49.033$; year: 1998; individualCount: 1 ; sex: female; catalogNumber: SMF 80033; recordedBy: Edgar Lehr leg.; institutionID: SMF

m. scientificName: Helicops angulatus (Linnaeus, 1758); country: Ecuador; stateProvince: Pastaza; locality: Arutam, km 48 Transamazonica; verbatimLocality: 880; verbatimLatitude: $-^{\circ} 47.07$; verbatimLongitude: $-77^{\circ} 49.96$; year: 1996 ; individualCount: 1 ; 
sex: female; catalogNumber: SMF 90947; recordedBy: Gunther Köhler, R. Seipp, S. Moya leg.; institutionID: SMF

n. scientificName: Helicops angulatus (Linnaeus, 1758); individualCount: 1; sex: male; catalogNumber: SMF 91832; institutionID: SMF

o. $\quad$ scientificName: Helicops angulatus (Linnaeus, 1758); individualCount: 1; sex: female; catalogNumber: SMF 91833; institutionID: SMF

p. $\quad$ scientificName: Helicops angulatus (Linnaeus, 1758); individualCount: 1; sex: female; catalogNumber: SMF 91834; institutionID: SMF

q. $\quad$ scientificName: Helicops angulatus (Linnaeus, 1758); country: Suriname; year: 1893; individualCount: 1; sex: female; catalogNumber: SMNS 13438; recordedBy: Hartmann leg.; institutionID: SMF

r. ScientificName: Helicops angulatus (Linnaeus, 1758); country: Brazil; stateProvince: Bahia; year: 1854; individualCount: 1; sex: female; catalogNumber: SMNS 3063; recordedBy: F. Glocker leg.; institutionID: SMNS

s. $\quad$ scientificName: Helicops angulatus (Linnaeus, 1758); year: 1843; individualCount: 1; sex: female; catalogNumber: SMNS 3064.1; recordedBy: A. Kappler; institutionID: SMNS

t. $\quad$ scientificName: Helicops angulatus (Linnaeus, 1758); individualCount: 1; sex: male; catalogNumber: SMNS 3064.2; institutionID: SMNS

u. $\quad$ scientificName: Helicops angulatus (Linnaeus, 1758); year: 1985; individualCount: 1; sex: male; catalogNumber: SMNS 6394; recordedBy: A. Schlüter leg.; institutionID: SMNS

v. ScientificName: Helicops angulatus (Linnaeus, 1758); country: Guyana; stateProvince: unknown province; locality: Roraima-Gebiet; individualCount: 1; sex: female; catalogNumber: ZFMK 47670; institutionID: ZFMK

w. ScientificName: Helicops angulatus (Linnaeus, 1758); country: Brazil; stateProvince: Amazonas; locality: Jurua; individualCount: 1; sex: male; catalogNumber: ZFMK 8403; institutionID: ZFMK

x. ScientificName: Helicops angulatus (Linnaeus, 1758); country: Brazil; stateProvince: Amazonas; locality: Jurua; individualCount: 1; sex: female; catalogNumber: ZFMK 8404; institutionID: ZFMK

y. $\quad$ scientificName: Helicops angulatus (Linnaeus, 1758); country: Brazil; stateProvince: Maranhao; individualCount: 1; sex: male; catalogNumber: ZMB 10854; recordedBy: S. Eye leg.; institutionID: ZMB

z. $\quad$ scientificName: Helicops angulatus (Linnaeus, 1758); individualCount: 1; sex: female; catalogNumber: ZMB 2303; recordedBy: M. Bloch leg.; institutionID: ZMB

aa. $\quad$ scientificName: Helicops angulatus (Linnaeus, 1758); country: French Guiana; individualCount: 1; sex: female; catalogNumber: ZMB 2305; institutionID: ZMB

ab. ScientificName: Helicops angulatus (Linnaeus, 1758); country: Suriname; stateProvince: Paramaribo; locality: Paramaribo; individualCount: 1; sex: female; catalogNumber: ZMB 25975A; recordedBy: K. Heller leg.; institutionID: ZMB

ac. $\quad$ scientificName: Helicops angulatus (Linnaeus, 1758); country: Suriname; stateProvince; Paramaribo; locality: Paramaribo; individualCount: 1; sex: male; catalogNumber: ZMB 25975B; recordedBy: K. Heller leg.; institutionID: ZMB

ad. ScientificName: Helicops angulatus (Linnaeus, 1758); country: Suriname; stateProvince: Paramaribo; locality: Paramaribo; individualCount: 1; sex: male; catalogNumber: ZMB 26382; recordedBy: K. Heller leg.; institutionID: ZMB

ae. $\quad$ scientificName: Helicops angulatus (Linnaeus, 1758); country: Brazil; individualCount: 1; sex: male; catalogNumber: ZMB 27783; recordedBy: Aq. Zoo. leg.; institutionID: ZMB

af. ScientificName: Helicops angulatus (Linnaeus, 1758); country: Brazil; stateProvince: Para; locality: Rio Caramarapy; individualCount: 1; sex: male; catalogNumber: ZMB 47771; recordedBy: K. Lako leg.; institutionID: ZMB 
ag. $\quad$ scientificName: Helicops angulatus (Linnaeus, 1758); country: French Guiana; individualCount: 1; sex: female; catalogNumber: ZMB 54167; institutionID: ZMB

ah. $\quad$ scientificName: Helicops angulatus (Linnaeus, 1758); individualCount: 1; sex: female; catalogNumber: ZMB 64697; recordedBy: Anat. Sammlung leg.; institutionID: ZMB

ai. $\quad$ scientificName: Helicops angulatus (Linnaeus, 1758); individualCount: 1; sex: female; catalogNumber: ZMB 64698; recordedBy: Anat. Sammlung leg.; institutionID: ZMB

aj. $\quad$ scientificName: Helicops angulatus (Linnaeus, 1758); individualCount: 1; sex: female; catalogNumber: ZMB 89648; institutionID: ZMB

ak. $\quad$ scientificName: Helicops angulatus (Linnaeus, 1758); individualCount: 1; sex: female; catalogNumber: ZMB 89649; institutionID: ZMB

al. $\quad$ scientificName: Helicops angulatus (Linnaeus, 1758); country: Suriname; year: <1858; individualCount: 1; sex: male; catalogNumber: ZSM 1525/0; institutionID: ZSM

am. $\quad$ scientificName: Helicops angulatus (Linnaeus, 1758); country: Brazil; year: <1907; individualCount: 1; sex: male; catalogNumber: ZSM 1526/0; institutionID: ZSM

an. ScientificName: Helicops angulatus (Linnaeus, 1758); country: Brazil; locality: in provinciae Bahiae adjacentibus; year: 1817-1820; individualCount: 1; sex: male; catalogNumber: ZSM 1528/0; recordedBy: Spix \& Martius leg.; institutionID: ZSM

ao. $\quad$ scientificName: Helicops angulatus (Linnaeus, 1758); country: Brazil; stateProvince: Pará; locality: Peixeboi (an der Bragançabahn); year: 1910; individualCount: 1; sex: female; catalogNumber: ZSM 247/1983; recordedBy: L. Müller leg.; institutionID: ZSM

ap. $\quad$ scientificName: Helicops angulatus (Linnaeus, 1758); country: Brazil; stateProvince: Pará; locality: Rio Branco bei Obidos; year: 1912; individualCount: 1; sex: female; catalogNumber: ZSM 264/2017; recordedBy: E. Snethlage leg.; institutionID: ZSM

aq. $\quad$ scientificName: Helicops angulatus (Linnaeus, 1758); country: Peru; stateProvince: Huánuco; locality: Biologische Station Panguana (unterer Rio Yuyapichis, ca. 140 km SSW Pucallpa); year: 1982; individualCount: 1; sex: female; catalogNumber: ZSM 37/2015; recordedBy: E.-G. Burmeister leg.; institutionID: ZSM

ar. $\quad$ scientificName: Helicops angulatus (Linnaeus, 1758); country: Bolivia; stateProvince: Chapare; year: 1949; individualCount: 1; sex: male; catalogNumber: ZSM 516/2003; recordedBy: R. Zischka leg.; institutionID: ZSM

as. $\quad$ scientificName: Helicops angulatus (Linnaeus, 1758); country: Columbia; stateProvince: Guainía; locality: Inirida; year: 1976; individualCount: 1; sex: male; catalogNumber: ZSM 518/2003; recordedBy: H. Herkner leg.; institutionID: ZSM

at. ScientificName: Helicops angulatus (Linnaeus, 1758); country: Peru; stateProvince: Ucayali; locality: Yarina Cocha bei Pucallpa; year: 1982; individualCount: 1; sex: female; catalogNumber: ZSM 59/1985; recordedBy: Maulhardt leg.; institutionID: ZSM

au. $\quad$ scientificName: Helicops angulatus (Linnaeus, 1758); country: Brazil; stateProvince: Sao Paulo; locality: Puerto Tiberica am Rio Parana [= Porto Tibirica]; year: 1938; individualCount: 1; sex: male; catalogNumber: ZSM 595/2003; recordedBy: O. Schindler leg.; institutionID: ZSM

\section{Diagnosis}

Helicops angulatus can be distinguished from all its congeners except $H$. scalaris and $H$. apiaka by having subcaudal keels, 17-20 dorsal scale rows at midbody, and 103123 ventrals. From $H$. scalaris it differs in having no intergenials (for information on references see Suppl. material 3; for summarized pholidosis information of the examined speciemens see Table 1). For differentiation from $H$. apiaka see identification of $H$. apiaka. 


\section{Distribution}

The distribution of $H$. angulatus extends over nearly the complete northern part of South America. As shown in Fig. 1a, the distribution range extends from Columbia to the east coast of Brazil and from Venezuela and offshore islands to the Brazilian province Sao Paulo.

\section{Helicops apiaka Kawashita-Ribeiro et al., 2013}

\section{Diagnosis}

According to the information given in Kawashita-Ribeiro et al. (2013), H. apiaka can be distinguished from all its congeners except $H$. angulatus and $H$. hagmanni by the following combination of characteristics: absent intergenials, subcaudal keels present, and 21-22 dorsal scale rows at midbody. From H. hagmanni it differs in having 79-103 subcaudal scales (versus $50-59$ in $H$. hagmanni). From $H$. angulatus it differs by having 21-24 dorsal scale rows at anterior body, 21-22 at midbody and 17-19 at posterior body (versus 19-21/19-20/17-19 in H. angulatus) and by having 118-127 ventral scales in males and 124-132 in females (versus 105-123 in male and 109-123 in female $H$. angulatus) as well as hemipenes morphology. The specimens examined in this study originated from neighbouring areas to the $H$. apiaka locations. Our own examinations revealed that males of $H$. angulatus possess 103-119 ventrals in males and 104-125 in females. There is considerable overlap especially between female specimens of the two species. This would exclude this character as identification character. In addition to that we came across two specimens having the morphology of $H$. angulatus but showing considerably more ventrals than usual (SMF 17819, a female, with 156 ventrals and ZSM 0595/2003, female, with 130 ventrals). There is no locality information for SMF 17819. ZSM 0595/2003 was collected at the Rio Parana in Porto Tibiriça, Sao Paulo, Brazil. This is approximately $1300 \mathrm{~km}$ apart from the distribution range of $H$. apiaka. The distribution area of $H$. apiaka probably presents important information for the distinguishing of the two species, since the variation throughout the whole distribution range of $H$. angulatus might be considerably bigger than the variation of the populations neighbouring $H$. apiaka populations (for information on references see Suppl. material 3).

\section{Distribution}

The only known specimens of $H$. apiaka are from northern Mato Grosso and southern Pará (Fig. 1b).

\section{Morphology remark}

Moraes-da-Silva et al. (2019) states that $H$. apiaka has 19-21 dorsal scale rows at midbody, which would eliminate this character as a diagnostic character to distinguish it from $H$. angulatus. This would leave only the number of ventrals in females as a 
diagnostic trait. However, the before mentioned publication examined the same specimens for $\mathrm{H}$. apiaka as in the original species description (Kawashita-Ribeiro et al. 2013). Therefore, at this point we trust the data given in the original species description.

Helicops boitata Moraes-da-Silva, Amaro, Sales-Nunes, Strüssmann, Teixeira, Andrade, Sudré, Recoder, Rodrigues, Curcio, 2019

\section{Diagnosis}

Helicops boitata differs from all its congeners by the presence of an entire nasal scale and 25 dorsal scale rows at midbody, reducing to 21 anterior to cloaca (for information on references see Suppl. material 3).

\section{Distribution}

Helicops boitata is only known from the Pantanal at Transpantaneira road in the municipality of Pocone, Mato Grosso, Brazil (Fig. 1c).

\section{Helicops carinicaudus (Wied-Neuwied, 1825)}

\section{Materials}

a. $\quad$ scientificName: Helicops carinicaudus (Wied-Neuwied, 1825); country: Brazil; individualCount: 1; sex: male; catalogNumber: MTKD 15295; recordedBy: Fritzsche leg.; institutionID: MTKD

b. $\quad$ scientificName: Helicops carinicaudus (Wied-Neuwied, 1825); country: Brazil; individualCount: 1; sex: male; catalogNumber: MTKD 15505; institutionID: MTKD

c. $\quad$ scientificName: Helicops carinicaudus (Wied-Neuwied, 1825); country: Brazil; stateProvince: Rio Grande do Sul; locality: Rio Grande; year: 1886; individualCount: 1; sex: female; catalogNumber: SMF 17799; recordedBy: H. Ihering leg.; institutionID: SMF

d. $\quad$ scientificName: Helicops carinicaudus (Wied-Neuwied, 1825); country: Brazil; year: 1838; individualCount: 1; sex: male; catalogNumber: SMF 17800; recordedBy: C.v.Heyden leg.; institutionID: SMF

e. $\quad$ scientificName: Helicops carinicaudus (Wied-Neuwied, 1825); country: Brazil; stateProvince: Pernambuco; year: 1913-1914; individualCount: 1; sex: female; catalogNumber: SMF 34035; recordedBy: E. Bresslau leg.; institutionID: SMF

f. ScientificName: Helicops carinicaudus (Wied-Neuwied, 1825); country: Brazil; stateProvince: Rio Grande do Sul; locality: Porto Alegre; year: 1935; individualCount: 1; sex: female; catalogNumber: SMF 37925; recordedBy: A. Adolff leg.; institutionID: SMF

g. scientificName: Helicops carinicaudus (Wied-Neuwied, 1825); country: Brazil; stateProvince: Sao Paulo; locality: Umgebung von Sao Paulo; year: 1955; individualCount: 1; sex: male; catalogNumber: SMF 49723; recordedBy: M. Schetty leg.; institutionID: SMF

h. $\quad$ scientificName: Helicops carinicaudus (Wied-Neuwied, 1825); country: Brazil; stateProvince: Sao Paulo; locality: Rinopolis; year: 1954; individualCount: 1; sex: female; catalogNumber: SMF 51208; recordedBy: R. Mertens leg.; institutionID: SMF 
i. $\quad$ scientificName: Helicops carinicaudus (Wied-Neuwied, 1825); country: Argentina; stateProvince: Buenos Aires; locality: Punta Lara; individualCount: 1; sex: female; catalogNumber: ZFMK 30350; institutionID: ZFMK

j. $\quad$ scientificName: Helicops carinicaudus (Wied-Neuwied, 1825); individualCount: 1; sex: female; catalogNumber: ZMB 2296; institutionID: ZMB

k. $\quad$ scientificName: Helicops carinicaudus (Wied-Neuwied, 1825); country: Brazil; individualCount: 1; sex: female; catalogNumber: ZMB 2298; recordedBy: I. v. Olfers leg.; institutionID: ZMB

I. scientificName: Helicops carinicaudus (Wied-Neuwied, 1825); country: Brazil; year: $<1907$; individualCount: 1; sex: female; catalogNumber: ZSM 2585/0; institutionID: ZSM

\section{Diagnosis}

Helicops carinicaudus can be distinguished from all its congeners except $H$. danieli, $H$. infrataeniatus, $H$. leopardinus and $H$. phantasma by the following combination of characteristics: $17-19$ dorsal scale rows at midbody, reducing to 17 anterior to cloaca, 128-141 ventrals in males, and 128-148 ventrals in females. From $H$. danieli and $H$. leopardinus it differs in having a striped or uniform dorsum, versus blotched pattern in $H$. danieli and $H$. leopardinus. From $H$. infrataeniatus and $H$. phantasma it differs in having a yellow or cream venter with two series of black semilunar marks, between these, small, irregular black spots (for information on references see Suppl. material 3).

\section{Distribution}

The distribution of $H$. carinicaudus extends from the estuary of the Rio de La Plata along the shoreline of Brazil to the province Pernambuco (Fig. 1d).

\section{Helicops danieli Amaral, 1937}

\section{Materials}

a. $\quad$ scientificName: Helicops danieli Amaral, 1937; country: Columbia; stateProvince: Barranquilla; year: 1958; individualCount: 1; sex: female; catalogNumber: SMF 55074; recordedBy: A. Werner leg.; institutionID: SMF

b. $\quad$ scientificName: Helicops danieli Amaral, 1937; country: Columbia; stateProvince: Barranquilla; year: 1958; individualCount: 1; sex: female; catalogNumber: SMF 55115; recordedBy: A. Werner leg.; institutionID: SMF

c. ScientificName: Helicops danieli Amaral, 1937; country: Columbia; stateProvince: Barranquilla; year: 1958; individualCount: 1; sex: female; catalogNumber: SMF 55695; recordedBy: A. Werner leg.; institutionID: SMF

d. $\quad$ scientificName: Helicops danieli Amaral, 1937; country: Brazil; individualCount: 1; sex: male; catalogNumber: ZMB 9490; institutionID: ZMB

e. $\quad$ scientificName: Helicops danieli Amaral, 1937; country: Columbia; stateProvince: Bolivar; locality: Jesus del Rio; year: 1937; individualCount: 1; sex: female; catalogNumber: ZSM 596/2003; recordedBy: W. Hellmich leg.; institutionID: ZSM 


\section{Diagnosis}

Helicops danieli is readily distinguished from its congeners by its spotted dorsum in combination with a ventral pattern consisting of 2 rows of semilunar marks on a light background (for information on references see Suppl. material 3).

\section{Distribution}

Helicops danieli is only present in Colombia. In Colombia it seems to range mainly west of the Andes. There is a report from the lowland in the east near the Brazilian border (Yuki and Castano 1998, Fig. 1e). Specimen ZMB 9490 has the country-level locality Brazil without a precise locality given.

\section{Helicops gomesi Amaral, 1921}

\section{Diagnosis}

Helicops gomesi is distinguished from all its congeners except $H$. angulatus by having subcaudal keels, no intergenials, and 19 dorsal scale rows throughout its body. From $H$. angulatus it differs in having 125-132 ventrals in males and 128-132 in females (for information on references see Suppl. material 3).

\section{Distribution}

The distribution of $H$. gomesi extends from the Brazilian province Sao Paulo to the provinces Mato Grosso, Mato Grosso do Sul and Goias (Fig. 1f).

\section{Helicops hagmanni Roux, 1910}

\section{Materials}

a. ScientificName: Helicops hagmanni Roux, 1910; country: Brazil; stateProvince: Amazonas; year: 1831; individualCount: 1; sex: male; catalogNumber: MTKD 7801; recordedBy: Poeppig leg.; institutionID: MTKD

b. $\quad$ scientificName: Helicops hagmanni Roux, 1910; country: Brazil; stateProvince: Para; locality: Umgebung von Para; individualCount: 1; sex: female; catalogNumber: ZMB C-826; recordedBy: A. Freiherr v. Dungern leg.; institutionID: ZMB

\section{Diagnosis}

Helicops hagmanni is distinguished from all its congeners by having subcaudal keels, 21-29 dorsal scale rows at midbody, and 50-59 subcaudals (for information on references see Suppl. material 3). 


\section{Distribution}

The distribution of $H$. hagmanni ranges from the estuary of the Amazonas to the Brazilian province Amazonas and the Venezuelan province Amazonas. There is also one record from northern Rondônia (Silva Jr 1993, Fig. 2a).

\section{Morphologic remark}

The examined specimens had smooth subcaudal scales on the anterior part of the tail, changing to weakly keeled scales at posterior tail (see also Table 1).

\section{Helicops infrataeniatus Jan, 1865}

\section{Materials}

a. $\quad$ scientificName: Helicops infrataeniatus Jan, 1865; country: Argentina; stateProvince: Entre Rios; individualCount: 1; sex: female; catalogNumber: MTKD 29826; recordedBy: Strauss-Hiller leg.; institutionID: MTKD

b. $\quad$ scientificName: Helicops infrataeniatus Jan, 1865; country: Brazil; year: 1898; individualCount: 1; sex: female; catalogNumber: SMF 17795; recordedBy: P. Werner leg.; institutionID: SMF

c. scientificName: Helicops infrataeniatus Jan, 1865; country: Brazil; stateProvince: Parana; locality: Curityba; year: 1905; individualCount: 1; sex: female; catalogNumber: SMF 17796; recordedBy: A. Haas leg.; institutionID: SMF

d. ScientificName: Helicops infrataeniatus Jan, 1865; country: Brazil; stateProvince: Parana; locality: Curityba; year: 1905; individualCount: 1; sex: female; catalogNumber: SMF 17797; recordedBy: A. Haas leg.; institutionID: SMF

e. $\quad$ scientificName: Helicops infrataeniatus Jan, 1865; country: Brazil; stateProvince: Rio Grande do Sul; year: 1888; individualCount: 1; sex: female; catalogNumber: SMF 17801; recordedBy: $\mathrm{H}$. Ihering leg.; institutionID: SMF

f. scientificName: Helicops infrataeniatus Jan, 1865; country: Brazil; stateProvince: Rio Grande do Sul; locality: Espumoso Via Carasinho; year: 1954; individualCount: 1; sex: male; catalogNumber: SMF 51209; recordedBy: R. Mertens leg.; institutionID: SMF

g. ScientificName: Helicops infrataeniatus Jan, 1865; country: Brazil; stateProvince: Rio Grande do Sul; locality: Espumoso Via Carasinho; year: 1954; individualCount: 1; sex: female; catalogNumber: SMF 51210; recordedBy: R. Mertens leg.; institutionID: SMF

h. ScientificName: Helicops infrataeniatus Jan, 1865; country: Argentina; stateProvince: Chaco; locality: Roque Saenz Pena; year: 1965; individualCount: 1; sex: male; catalogNumber: SMF 67327; recordedBy: Foerster leg.; institutionID: SMF

i. $\quad$ scientificName: Helicops infrataeniatus Jan, 1865; country: Brazil; year: 1889; individualCount: 1; sex: female; catalogNumber: SMNS 3065; recordedBy: Umlauff leg.; institutionID: SMNS

j. ScientificName: Helicops infrataeniatus Jan, 1865; country: Brazil; stateProvince: Rio Grande do Sul; locality: Cachoeira do Sul; year: 1995; individualCount: 1; sex: female; catalogNumber: SMNS 9038; recordedBy: A. Kwet leg.; institutionID: SMNS

k. ScientificName: Helicops infrataeniatus Jan, 1865; country: Brazil; stateProvince: Rio Grande do Sul; locality: Sao Leopoldo; individualCount: 1; sex: female; catalogNumber: ZFMK 102469; recordedBy: leg.; institutionID: SMNS 
I. scientificName: Helicops infrataeniatus Jan, 1865; country: Argentina; stateProvince: Buenos Aires; locality: Punta Lara; individualCount: 1; sex: female; catalogNumber: ZFMK 102499; institutionID: ZFMK

m. scientificName: Helicops infrataeniatus Jan, 1865; individualCount: 1; sex: female; catalogNumber: ZFMK 102500; institutionID: ZFMK

n. $\quad$ scientificName: Helicops infrataeniatus Jan, 1865; individualCount: 1; sex: female; catalog Number: ZFMK 102501; institutionID: ZFMK

o. $\quad$ scientificName: Helicops infrataeniatus Jan, 1865; individualCount: 1; sex: female; catalogNumber: ZFMK 102502; institutionID: ZFMK

p. $\quad$ scientificName: Helicops infrataeniatus Jan, 1865; individualCount: 1; sex: male; catalogNumber: ZFMK 102503; institutionID: ZFMK

q. $\quad$ scientificName: Helicops infrataeniatus Jan, 1865; individualCount: 1; sex: female; catalogNumber: ZFMK 102504; institutionID: ZFMK

r. $\quad$ scientificName: Helicops infrataeniatus Jan, 1865; individualCount: 1; sex: male; catalogNumber: ZFMK 102505; institutionID: ZFMK

s. $\quad$ scientificName: Helicops infrataeniatus Jan, 1865; individualCount: 1; sex: female; catalog Number: ZFMK 102506; institutionID: ZFMK

t. scientificName: Helicops infrataeniatus Jan, 1865; individualCount: 1; sex: female; catalogNumber: ZFMK 102507; institutionID: ZFMK

u. $\quad$ scientificName: Helicops infrataeniatus Jan, 1865; individualCount: 1; sex: female; catalogNumber: ZFMK 102508; institutionID: ZFMK

v. ScientificName: Helicops infrataeniatus Jan, 1865; individualCount: 1; sex: female; catalogNumber: ZFMK 102509; institutionID: ZFMK

w. ScientificName: Helicops infrataeniatus Jan, 1865; country: Brazil; stateProvince: Rio Grande do Sul; locality: Campo Bom (wahrscheinlich); individualCount: 1; sex: female; catalogNumber: ZFMK 102630; institutionID: ZFMK

x. scientificName: Helicops infrataeniatus Jan, 1865; country: Brazil; individualCount: 1; sex: female; catalogNumber: ZMB 16436; recordedBy: Mücke leg.; institutionID: ZMB

y. $\quad$ scientificName: Helicops infrataeniatus Jan, 1865; country: Brazil; individualCount: 1; sex: male; catalogNumber: ZMB 16437; recordedBy: Mücke leg.; institutionID: ZMB

z. scientificName: Helicops infrataeniatus Jan, 1865; country: Brazil; individualCount: 1; sex: female; catalog Number: ZMB 16438; recordedBy: Mücke leg.; institutionID: ZMB

aa. scientificName: Helicops infrataeniatus Jan, 1865; country: Argentina; individualCount: 1; sex: male; catalogNumber: ZMB 20606A; recordedBy: R. Hauthal leg.; institutionID: ZMB

ab. $\quad$ scientificName: Helicops infrataeniatus Jan, 1865; country: Argentina; individualCount: 1; sex: female; catalogNumber: ZMB 20606B; recordedBy: R. Hauthal leg.; institutionID: ZMB

ac. $\quad$ scientificName: Helicops infrataeniatus Jan, 1865; country: Argentina; individualCount: 1; sex: female; catalogNumber: ZMB 20606C; recordedBy: R. Hauthal leg.; institutionID: ZMB

ad. $\quad$ scientificName: Helicops infrataeniatus Jan, 1865; country: Argentina; individualCount: 1; sex: male; catalogNumber: ZMB 20607; recordedBy: R. Hauthal leg.; institutionID: ZMB

ae. $\quad$ scientificName: Helicops infrataeniatus Jan, 1865; country: Argentina; individualCount: 1; sex: male; catalogNumber: ZMB 20608A; recordedBy: R. Hauthal leg.; institutionID: ZMB

af. $\quad$ scientificName: Helicops infrataeniatus Jan, 1865; country: Argentina; individualCount: 1; sex: male; catalogNumber: ZMB 20608B; recordedBy: R. Hauthal leg.; institutionID: ZMB

ag. $\quad$ scientificName: Helicops infrataeniatus Jan, 1865; country: Argentina; individualCount: 1; sex: female; catalogNumber: ZMB 20608C; recordedBy: R. Hauthal leg.; institutionID: ZMB 
ah. $\quad$ scientificName: Helicops infrataeniatus Jan, 1865; country: Argentina; individualCount: 1; sex: male; catalogNumber: ZMB 20609A; recordedBy: R. Hauthal leg.; institutionID: ZMB

ai. $\quad$ scientificName: Helicops infrataeniatus Jan, 1865; country: Argentina; individualCount: 1; sex: female; catalogNumber: ZMB 20609B; recordedBy: R. Hauthal leg.; institutionID: ZMB

aj. $\quad$ scientificName: Helicops infrataeniatus Jan, 1865; country: Argentina; individualCount: 1; sex: male; catalogNumber: ZMB 20609C; recordedBy: R. Hauthal leg.; institutionID: ZMB

ak. $\quad$ scientificName: Helicops infrataeniatus Jan, 1865; individualCount: 1; sex: female; catalogNumber: ZMB 20610A; institutionID: ZMB

al. $\quad$ scientificName: Helicops infrataeniatus Jan, 1865; individualCount: 1; sex: female; catalogNumber: ZMB 20610B; institutionID: ZMB

am. ScientificName: Helicops infrataeniatus Jan, 1865; country: Argentina; individualCount: 1; sex: male; catalogNumber: ZMB 20613A; recordedBy: R. Hauthal leg.; institutionID: ZMB

an. ScientificName: Helicops infrataeniatus Jan, 1865; country: Argentina; individualCount: 1; sex: female; catalogNumber: ZMB 20613B; recordedBy: R. Hauthal leg.; institutionID: ZMB

ao. $\quad$ scientificName: Helicops infrataeniatus Jan, 1865; country: Argentina; individualCount: 1; sex: female; catalogNumber: ZMB 20613C; recordedBy: R. Hauthal leg.; institutionID: ZMB

ap. $\quad$ scientificName: Helicops infrataeniatus Jan, 1865; country: Argentina; individualCount: 1; sex: female; catalogNumber: ZMB 20613D; recordedBy: R. Hauthal leg.; institutionID: ZMB

aq. $\quad$ scientificName: Helicops infrataeniatus Jan, 1865; country: Argentina; individualCount: 1; sex: female; catalogNumber: ZMB 20613E; recordedBy: R. Hauthal leg.; institutionID: ZMB

ar. $\quad$ scientificName: Helicops infrataeniatus Jan, 1865; country: Argentina; individualCount: 1; sex: female; catalogNumber: ZMB 20613F; recordedBy: R. Hauthal leg.; institutionID: ZMB

as. $\quad$ scientificName: Helicops infrataeniatus Jan, 1865; country: Brazil; stateProvince: Rio Grande do Sul; locality: Porto Alegre; individualCount: 1; sex: male; catalogNumber: ZMB 6373; recordedBy: R. Hensel leg.; institutionID: ZMB

at. $\quad$ scientificName: Helicops infrataeniatus Jan, 1865; country: Brazil; stateProvince: Rio Grande do Sul; locality: Porto Alegre; individualCount: 1; sex: female; catalogNumber: ZMB 6840A; recordedBy: R. Hensel leg.; institutionID: ZMB

au. $\quad$ scientificName: Helicops infrataeniatus Jan, 1865; country: Brazil; stateProvince: Rio Grande do Sul; locality: Porto Alegre; individualCount: 1; sex: female; catalogNumber: ZMB 6840B; recordedBy: R. Hensel leg.; institutionID: ZMB

av. $\quad$ scientificName: Helicops infrataeniatus Jan, 1865; individualCount: 1; sex: female; catalogNumber: ZMB 79245; institutionID: ZMB

aw. scientificName: Helicops infrataeniatus Jan, 1865; individualCount: 1; sex: female; catalogNumber: ZMB 79246; institutionID: ZMB

ax. $\quad$ scientificName: Helicops infrataeniatus Jan, 1865; individualCount: 1; sex: male; catalogNumber: ZMB 79247; institutionID: ZMB

ay. $\quad$ scientificName: Helicops infrataeniatus Jan, 1865; individualCount: 1; sex: male; catalogNumber: ZMB 79248; institutionID: ZMB

az. $\quad$ scientificName: Helicops infrataeniatus Jan, 1865; country: Brazil; stateProvince: Rio Grande do Sul; locality: Porto Alegre; individualCount: 1; sex: male; catalogNumber: ZMB 79249; recordedBy: R. Hensel leg.; institutionID: ZMB 
ba. scientificName: Helicops infrataeniatus Jan, 1865; country: Brazil; stateProvince: Rio Grande do Sul; locality: Porto Alegre; individualCount: 1; sex: male; catalogNumber: ZMB 79250; recordedBy: R. Hensel leg.; institutionID: ZMB

bb. $\quad$ scientificName: Helicops infrataeniatus Jan, 1865; individualCount: 1; sex: male; catalogNumber: ZMB 89646; institutionID: ZMB

bc. $\quad$ scientificName: Helicops infrataeniatus Jan, 1865; individualCount: 1; sex: male; catalogNumber: ZMB 89647; institutionID: ZMB

\section{Diagnosis}

This species can be distinguished from all other congeners except $H$. carinicaudus, $H$. nentur and $H$. tapajonicus by the combination of 17-20 dorsal scale rows at midbody, and a uniform or longitudinally striped dorsum. From $H$. nentur it differs in having a semidivided nasal scale, whereas $H$. nentur has an entire nasal scale. From $H$. carinicaudus it differs in having a cream or red venter with 1-3 dark stripes or darkly checkered or black with light spots. Sometimes the patterns shift into each other. From $H$. tapajonicus it differs in having strongly keeled dorsal scales, whereas $H$. tapajonicus has only a weak dorsal keeling. Additionally, $H$. tapajonicus possesses a ventrolateral greenish stripe, which is absent in $H$. infrataeniatus (for information on references see Suppl. material 3). Furthermore, these two species have allopatric distribution ranges.

\section{Distribution}

Helicops infrataeniatus is present in the southern Brazilian states Mato Grosso do Sul, Sao Paulo, Parana, Santa Catarina, Rio Grande do Sul and northwest Argentina. For Uruguay Carreira Vidal et al. (2005) described the presence of $H$. infrataeniatus from the north of the department of Rocha through the department of Cerro Largo continuing to the north of the country, being frequent in the departments of Artigas and Salto (Fig. $2 b)$.

\section{Morphology remark}

Kawashita-Ribeiro et al. (2013) reported subcaudal keels in $H$. infrataeniatus. In contrast, 57 of the 58 examined specimens during this study showed smooth subcaudal scales. Only SMNS 3065 had subcaudal keels (see Table 1).

\section{Helicop leopardinus (Schlegel, 1837)}

\section{Materials}

a. ScientificName: Helicops leopardinus (Schlegel, 1837); country: Columbia; individualCount: 1; sex: female; catalogNumber: MTKD 15506; institutionID: MTKD

b. ScientificName: Helicops leopardinus (Schlegel, 1837); country: Argentina; stateProvince: Cordoba; locality: San Franciso; individualCount: 1; sex: female; catalogNumber: MTKD 27443; recordedBy: Strauss-Hiller leg.; institutionID: MTKD

c. ScientificName: Helicops leopardinus (Schlegel, 1837); country: Argentina; stateProvince: Cordoba; locality: San Franciso; individualCount: 1; sex: female; catalogNumber: MTKD 28115; recordedBy: Strauss-Hiller leg.; institutionID: MTKD 
d. ScientificName: Helicops leopardinus (Schlegel, 1837); country: Argentina; locality: Central Chaco; individualCount: 1; sex: male; catalogNumber: MTKD 28716; recordedBy: Strauss-Hiller leg.; institutionID: MTKD

e. $\quad$ scientificName: Helicops leopardinus (Schlegel, 1837); country: Argentina; stateProvince: Chaco; locality: Resistencia; individualCount: 1; sex: female; catalogNumber: MTKD 29825; recordedBy: Strauss-Hiller leg.; institutionID: MTKD

f. ScientificName: Helicops leopardinus (Schlegel, 1837); country: Bolivia; stateProvince: Santa Cruz; locality: Velasco, Campamento; verbatimLocality: 185; verbatimLatitude: $-15^{\circ} 10.493$; verbatimLongitude: $-61^{\circ} 0.968$; year: 2007 ; individualCount: 1 ; sex: female; catalogNumber: SMF 100015; recordedBy: M. Jansen leg.; institutionID: SMF

g. scientificName: Helicops leopardinus (Schlegel, 1837); country: Brazil; stateProvince: Mato Grosso; locality: Cujapa; year: 1885; individualCount: 1; sex: female; catalogNumber: SMF 17807; recordedBy: I. Schumacher leg.; institutionID: SMF

h. $\quad$ scientificName: Helicops leopardinus (Schlegel, 1837); country: Brazil; locality: Northern Brazil; year: 1897; individualCount: 1; sex: male; catalogNumber: SMF 17809; recordedBy: O. Boettger leg.; institutionID: SMF

i. $\quad$ scientificName: Helicops leopardinus (Schlegel, 1837); country: Paraguay; year: 1972; individualCount: 1; sex: female; catalogNumber: SMF 67860; recordedBy: M. Schetty leg.; institutionID: SMF

j. $\quad$ scientificName: Helicops leopardinus (Schlegel, 1837); country: Paraguay; year: 1972; individualCount: 1; sex: female; catalogNumber: SMF 67861; recordedBy: M. Schetty leg.; institutionID: SMF

k. $\quad$ scientificName: Helicops leopardinus (Schlegel, 1837); country: Paraguay; year: 1972; individualCount: 1; sex: female; catalogNumber: SMF 67862; recordedBy: W.v.d. Wall leg.; institutionID: SMF

I. ScientificName: Helicops leopardinus (Schlegel, 1837); country: Brazil; individualCount: 1; sex: male; catalogNumber: ZFMK 36339; institutionID: ZFMK

m. scientificName: Helicops leopardinus (Schlegel, 1837); country: Paraguay; stateProvince: Asunción; locality: Pilcomayo; individualCount: 1; sex: female; catalogNumber: ZFMK 59774; institutionID: ZFMK

n. scientificName: Helicops leopardinus (Schlegel, 1837); country: Paraguay; stateProvince: Asunción; locality: Pilcomayo; individualCount: 1; sex: male; catalogNumber: ZFMK 59775; institutionID: ZFMK

o. ScientificName: Helicops leopardinus (Schlegel, 1837); country: Bolivia; stateProvince: Santa Cruz; locality: between Florida \& Meura, Rio Paraguay; individualCount: 1; sex: male; catalogNumber: ZFMK 60153; institutionID: ZFMK

p. scientificName: Helicops leopardinus (Schlegel, 1837); country: Bolivia; stateProvince: Beni; locality: Campamento Encanto; individualCount: 1; sex: female; catalogNumber: ZFMK 62836; institutionID: ZFMK

q. ScientificName: Helicops leopardinus (Schlegel, 1837); country: Paraguay; individualCount: 1; sex: male; catalogNumber: ZMB 10749; recordedBy: R. Rohde leg.; institutionID: ZFMK

r. ScientificName: Helicops leopardinus (Schlegel, 1837); country: Argentina; individualCount: 1; sex: female; catalogNumber: ZMB 20611A; recordedBy: R. Hauthal leg.; institutionID: ZMB

s. $\quad$ scientificName: Helicops leopardinus (Schlegel, 1837); country: Argentina; individualCount: 1; sex: female; catalogNumber: ZMB 20611B; recordedBy: R. Hauthal leg.; institutionID: ZMB 
t. ScientificName: Helicops leopardinus (Schlegel, 1837); country: Argentina; stateProvince: Salta; locality: Tartagal; individualCount: 1; sex: male; catalogNumber: ZMB 26040A; recordedBy: Neumayer leg.; institutionID: ZMB

u. scientificName: Helicops leopardinus (Schlegel, 1837); country: Argentina; stateProvince: Salta; locality: Tartagal; individualCount: 1; catalogNumber: ZMB 26040B; recordedBy: Neumayer leg.; institutionID: ZMB

v. ScientificName: Helicops leopardinus (Schlegel, 1837); country: Brazil; stateProvince: Bahia; individualCount: 1; sex: male; catalogNumber: ZMB 7545; recordedBy: O. Wucherer leg.; institutionID: ZMB

w. $\quad$ scientificName: Helicops leopardinus (Schlegel, 1837); individualCount: 1; sex: female; catalogNumber: ZMB 89665; institutionID: ZMB

X. scientificName: Helicops leopardinus (Schlegel, 1837); country: Paraguay; stateProvince: Alto Paraguguay; locality: Puerto Sastre; year: 1931; individualCount: 1; sex: female; catalogNumber: ZSM 1026/2010; recordedBy: G. Walter leg.; institutionID: ZMB scientificName: Helicops leopardinus (Schlegel, 1837); country: Paraguay; stateProvince: Alto Paraguguay; locality: Puerto Sastre; year: 1931; individualCount: 1; sex: male; catalogNumber: ZSM 1027/2010; recordedBy: G. Walter leg.; institutionID: ZSM

z. scientificName: Helicops leopardinus (Schlegel, 1837); country: Paraguay; stateProvince: Alto Paraguguay; locality: Puerto Sastre; year: 1931; individualCount: 1; sex: male; catalogNumber: ZSM 1028/2010; recordedBy: G. Walter leg.; institutionID: ZSM

aa. ScientificName: Helicops leopardinus (Schlegel, 1837); country: Paraguay; stateProvince: Alto Paraguguay; locality: Puerto Sastre; year: 1931; individualCount: 1; sex: female; catalogNumber: ZSM 1029/2010; recordedBy: G. Walter leg.; institutionID: ZSM

ab. scientificName: Helicops leopardinus (Schlegel, 1837); country: Paraguay; stateProvince: Alto Paraguguay; locality: Puerto Sastre; year: 1931; individualCount: 1; sex: female; catalogNumber: ZSM 1030/2010; recordedBy: G. Walter leg.; institutionID: ZSM

ac. $\quad$ scientificName: Helicops leopardinus (Schlegel, 1837); country: Paraguay; stateProvince: Alto Paraguguay; locality: Puerto Sastre; year: 1931; individualCount: 1; sex: female; catalogNumber: ZSM 1031/2010; recordedBy: G. Walter leg.; institutionID: ZSM

ad. ScientificName: Helicops leopardinus (Schlegel, 1837); country: Paraguay; stateProvince: Alto Paraguguay; locality: Puerto Sastre; year: 1931; individualCount: 1; sex: female; catalogNumber: ZSM 1032/2010; recordedBy: G. Walter leg.; institutionID: ZSM

ae. scientificName: Helicops leopardinus (Schlegel, 1837); country: Paraguay; stateProvince: Alto Paraguguay; locality: Puerto Sastre; year: 1931; individualCount: 1; sex: female; catalogNumber: ZSM 1033/2010; recordedBy: G. Walter leg.; institutionID: ZSM

af. $\quad$ scientificName: Helicops leopardinus (Schlegel, 1837); country: Paraguay; stateProvince: Alto Paraguguay; locality: Puerto Sastre; year: 1931; individualCount: 1; sex: female; catalogNumber: ZSM 1034/2010; recordedBy: G. Walter leg.; institutionID: ZSM

ag. ScientificName: Helicops leopardinus (Schlegel, 1837); country: Paraguay; stateProvince: Alto Paraguguay; locality: Puerto Sastre; year: 1931; individualCount: 1; sex: female; catalogNumber: ZSM 1035/2010; recordedBy: G. Walter leg.; institutionID: ZSM

ah. scientificName: Helicops leopardinus (Schlegel, 1837); country: Paraguay; stateProvince: Alto Paraguguay; locality: Puerto Sastre; year: 1931; individualCount: 1; sex: male; catalogNumber: ZSM 1036/2010; recordedBy: G. Walter leg.; institutionID: ZSM

ai. $\quad$ scientificName: Helicops leopardinus (Schlegel, 1837); country: Paraguay; stateProvince: Alto Paraguguay; locality: Puerto Sastre; year: 1931; individualCount: 1; sex: female; catalogNumber: ZSM 1037/2010; recordedBy: G. Walter leg.; institutionID: ZSM

aj. $\quad$ scientificName: Helicops leopardinus (Schlegel, 1837); country: Paraguay; stateProvince: Alto Paraguguay; locality: Puerto Sastre; year: 1931; individualCount: 1; sex: female; catalogNumber: ZSM 1038/2010; recordedBy: G. Walter leg.; institutionID: ZSM 
ak. $\quad$ scientificName: Helicops leopardinus (Schlegel, 1837); country: Paraguay; stateProvince: Alto Paraguguay; locality: Puerto Sastre; year: 1931; individualCount: 1; sex: female; catalogNumber: ZSM 1039/2010; recordedBy: G. Walter leg.; institutionID: ZSM

al. $\quad$ scientificName: Helicops leopardinus (Schlegel, 1837); country: Paraguay; stateProvince: Alto Paraguguay; locality: Puerto Sastre; year: 1931; individualCount: 1; sex: female; catalogNumber: ZSM 1040/2010; recordedBy: G. Walter leg.; institutionID: ZSM

am. ScientificName: Helicops leopardinus (Schlegel, 1837); country: Bolivia; stateProvince: Departamento Beni; locality: Rio Madre de Dios; year: <1923?; individualCount: 1; sex: female; catalogNumber: ZSM 134/1947; recordedBy: Zimmermann leg.; institutionID: ZSM

an. ScientificName: Helicops leopardinus (Schlegel, 1837); country: Brazil; year: 1817-1820; individualCount: 1; sex: male; catalogNumber: ZSM 1523/0; recordedBy: Spix \& Martius leg.; institutionID: ZSM

ao. $\quad$ scientificName: Helicops leopardinus (Schlegel, 1837); country: Bolivia; stateProvince: Santa Cruz; locality: San Fermin (100 km nördlich Puerto Suarez); year: 1926; individualCount: 1; sex: female; catalogNumber: ZSM 172/1929; recordedBy: I. Deutsche Chaco-Expedition leg.; institutionID: ZSM

ap. ScientificName: Helicops leopardinus (Schlegel, 1837); country: Argentina; stateProvince: Santa Fe; locality: Rosario de Santa Fe; year: 1903-1906; individualCount: 1; sex: female; catalogNumber: ZSM 268/2017; recordedBy: K. König leg.; institutionID: ZSM

aq. ScientificName: Helicops leopardinus (Schlegel, 1837); country: Argentina; stateProvince: Santa Fe; locality: Rosario de Santa Fe; year: 1907; individualCount: 1; sex: female; catalogNumber: ZSM 269/2017; recordedBy: von Stromer leg.; institutionID: ZSM

ar. ScientificName: Helicops leopardinus (Schlegel, 1837); country: Argentina; stateProvince: Santa Fe; locality: Rosario de Santa Fe; year: 1907; individualCount: 1; sex: female; catalogNumber: ZSM 270/2017; recordedBy: von Stromer leg.; institutionID: ZSM

\section{Diagnosis}

Helicops leopardinus is distinguished from all its congeners except $H$. danieli and $H$. gomesi by the combination of grayish-olive to grayish-brown dorsum with 4-5 series of alternating dark spots, 18-22 dorsal scale rows at midbody, and absent intergenials. From $H$. danieli it differs in having a cream, yellow or red venter, checkered or banded black or both (versus cream venter with two medial rows of black semilunar marks). From $H$. gomesi it differs in having no subcaudal keels (for information on references see Suppl. material 3).

\section{Distribution}

Helicops leopardinus is present from the northwest of Argentina to the estuary of the Amazon and from Ecuador to the Brazilian state Bahia. There are no records of this species in the central Amazon Basin (Fig. 2c).

\section{Morphology remark}

ZSM 134/1947, a female, possesses 109 subcaudals (versus 53-88 in females of $H$. leopardinus). We interpret this as an abnormality (see also Table 1). 


\section{Helicops modestus Günther, 1861}

\section{Materials}

a. ScientificName: Helicops modestus Günther, 1861; country: Brazil; stateProvince: Sao Paulo; year: 1881; individualCount: 1; sex: female; catalogNumber: SMF 17802; recordedBy: I. Duschanek leg.; institutionID: SMF

b. $\quad$ scientificName: Helicops modestus Günther, 1861; country: Brazil; stateProvince: Sao Paulo; year: 1881; individualCount: 1; sex: female; catalogNumber: SMF 17803; recordedBy: I. Duschanek leg.; institutionID: SMF

c. ScientificName: Helicops modestus Günther, 1861; country: Brazil; stateProvince: Sao Paulo; year: 1876; individualCount: 1; sex: female; catalogNumber: SMF 17804; recordedBy: C. Müller leg.; institutionID: SMF

d. ScientificName: Helicops modestus Günther, 1861; country: Brazil; stateProvince: Sao Paulo; year: 1876; individualCount: 1; sex: female; catalogNumber: SMF 17805; recordedBy: C. Müller leg.; institutionID: SMF

e. $\quad$ scientificName: Helicops modestus Günther, 1861; country: Brazil; stateProvince: Sao Paulo; individualCount: 1; sex: male; catalogNumber: SMF 17806; institutionID: SMF

f. ScientificName: Helicops modestus Günther, 1861; country: Brazil; stateProvince: Sao Paulo; locality: Umgebung von Sao Paulo; year: 1955; individualCount: 1; sex: female; catalogNumber: SMF 49724; recordedBy: M. Schetty leg.; institutionID: SMF

g. ScientificName: Helicops modestus Günther, 1861; country: Brazil; stateProvince: Sao Paulo; locality: Umgebung von Sao Paulo; year: 1955; individualCount: 1; sex: female; catalogNumber: SMF 49725; recordedBy: M. Schetty leg.; institutionID: SMF

h. $\quad$ scientificName: Helicops modestus Günther, 1861; country: Brazil; stateProvince: Sao Paulo; locality: Umgebung von Sao Paulo; year: 1955; individualCount: 1; sex: female; catalogNumber: SMF 49726; recordedBy: M. Schetty leg.; institutionID: SMF

i. ScientificName: Helicops modestus Günther, 1861; country: Brazil; stateProvince: Sao Paulo; locality: Umgebung von Sao Paulo; year: 1955; individualCount: 1; sex: female; catalogNumber: SMF 49727; recordedBy: M. Schetty leg.; institutionID: SMF

j. ScientificName: Helicops modestus Günther, 1861; country: Brazil; stateProvince: Sao Paulo; locality: Sao Miguel Paulista; year: 1955; individualCount: 1; sex: female; catalogNumber: SMF 51207; recordedBy: R. Mertens leg.; institutionID: SMF

k. ScientificName: Helicops modestus Günther, 1861; country: Brazil; stateProvince: Embu; locality: 25 km von Sao Paulo; year: 1954; individualCount: 1; sex: female; catalogNumber: SMF 91211; recordedBy: F. Weidinger leg.; institutionID: SMF

I. ScientificName: Helicops modestus Günther, 1861; country: Brazil; individualCount: 1; sex: male; catalogNumber: ZMB 2266; recordedBy: Reinhardt leg.; institutionID: ZMB

\section{Diagnosis}

Helicops modestus differs from all its congeners except $H$. carinicaudus, $H$. danieli, $H$. infrataeniatus, $H$. leopardinus and $H$. tapajonicus by having 19 dorsal scale rows at anterior body and 17-20 at midbody, and the absence of subcaudal keels. From the remaining species it differs in having a black to dark green dorsum with indistinct longitudinal stripes and a nearly uniform light cream venter, sometimes with faint flecks (for information on references see Suppl. material 3). 


\section{Distribution}

Helicops modestus is present from the Brazilian province Bahia to the province Paraná and seems to range from the east shore of Brazil to the south of Mato Grosso. There is also one specimen from the southern shore of Uruguay and one literature report from Volta Grande do Xingu in the Brazilian province Para, near its estuary into the Amazon (Vaz-Silva et al. 2015, Fig. 2d).

\section{Helicops nentur Costa, Santana, Leal, Koroiva \&Garcia, 2016}

\section{Diagnosis}

Helicops nentur differs from all its congeners by the combination of an entire nasal scale, and 17 dorsal scale rows at midbody (for information on references see Suppl. material 3).

\section{Distribution}

Helicops nentur is known only from the eastern half of the Brazilian Province Minas Gerais (Fig. 2e).

\section{Helicops pastazae Shreve, 1934}

\section{Material}

a. $\quad$ scientificName: Helicops pastazae Shreve, 1934; country: Ecuador; stateProvince: Napo; locality: Virgilio Davila (Borja), Quijos; year: <1952; individualCount: 1; sex: female; catalogNumber: ZSM 519/2003; recordedBy: J. Foerster leg.; institutionID: ZSM

\section{Diagnosis}

Helicops pastazae can be distinguished from all other congeners except $H$. hagmanni and $H$. yacu by the combination of having subcaudal keels, intergenials present, and 23-25 dorsal scale rows at midbody. From $H$. hagmanni it differs in having 72-117 subcaudal scales (versus 50-59 in $H$. hagmanni). From $H$. yacu it differs in having a semidivided nasal scale (entire in $\mathrm{H}$. yacu; for information on references see Suppl. material 3).

\section{Distribution}

Helicops pastazae is present in the northern part of Ecuador and the eastern part of Venezuela. There are no reports from Colombia (Fig. 2f). 


\section{Helicops petersi Rossman, 1976}

\section{Diagnosis}

Helicops petersi can be distinguished from all its congeners except $H$. pastazae by the combination of 21-23 dorsal scale rows at midbody, reducing to 16 anterior to cloaca, and 135-150 ventrals (for information on references see Suppl. material 3).

\section{Distribution}

Helicops petersi is known only from a very small area in the Ecuadorian province Napo (Fig. 3a).

\section{Helicops phantasma Moraes-da-Silva, Amaro, Nunes, Rodrigues \& Curcio, 2021}

\section{Diagnosis}

Helicops phantasma can be distinguished from all other congeners by having a dorsal pattern of dark spots fusing to irregular black blands, having 19/19/17-19 dorsal scale rows with moderate keels and hemipenial morphology (Moraes-da-Silva et al. 2021) (for information on references see Suppl. material 3).

\section{Distribution}

The species is only known from the Tocantins-Araguaia River Basin in the provinces Toncantins, Mato Grosso and Maranhão in northern Brazil (Moraes-da-Silva et al. 2021, Fig. 3b).

\section{Helicops polylepis Günther, 1861}

\section{Materials}

a. $\quad$ scientificName: Helicops polylepis Günther, 1861; country: Columbia; individualCount: 1; sex: female; catalogNumber: MTKD 15507; institutionID: MTKD

b. $\quad$ scientificName: Helicops polylepis Günther, 1861; country: Columbia; individualCount: 1; sex: female; catalogNumber: MTKD 15508; institutionID: MTKD

c. scientificName: Helicops polylepis Günther, 1861; country: Bolivia; stateProvince: Chaco; individualCount: 1; sex: female; catalogNumber: SMF 17821; recordedBy: F. Werner leg.; institutionID: SMF

d. ScientificName: Helicops polylepis Günther, 1861; country: Bolivia; individualCount: 1; sex: female; catalogNumber: SMF 17822; recordedBy: C.A. Hahn leg.; institutionID: SMF

e. $\quad$ scientificName: Helicops polylepis Günther, 1861; country: Brazil; stateProvince: Bahia; individualCount: 1; sex: male; catalogNumber: ZMB 17428; institutionID: ZMB 
f. ScientificName: Helicops polylepis Günther, 1861; country: Bolivia; stateProvince: La Paz; locality: La Paz; individualCount: 1; sex: male; catalogNumber: ZMB 26215; recordedBy: Stoecker leg.; institutionID: ZMB

g. scientificName: Helicops polylepis Günther, 1861; individualCount: 1; catalogNumber: ZMB 30993; institutionID: ZMB

\section{Diagnosis}

Helicops polylepis can be distinguished from all its congeners by the combination of 23-26 dorsal scale rows at midbody, the absence of intergenials, and a semidivided nasal scale (for information on references see Suppl. material 3).

\section{Distribution}

Helicops polylepis is present from southern Bolivia to the Amazon estuary and from southeastern Peru to the Brazilian province Bahia (Fig. 3c).

\section{Helicops scalaris Jan, 1865}

\section{Diagnosis}

This species can be distinguished from all its congeners except $H$. trivittatus by the combination of having intergenials, $110-119$ ventrals in males and 113-125 in females, and 67-95 subcaudals. From $H$. trivittatus it differs in having a blotched dorsum, versus striped in $H$. trivittatus (for information on references see Suppl. material 3).

\section{Distribution}

Helicops scalaris is known only from a small area in the northern border area between Colombia and Venezuela, western and northern of Lake Maracaibo in Venezuela (Fig. 3d).

\section{Helicops tapajonicus da Frota, 2005}

\section{Diagnosis}

This species can be distinguished from all its congeners by the combination of a uniform moss-green dorsum, laterally with a greenish-yellow stripe and a black and greenish-yellow banded venter (for information on references see Suppl. material 3).

\section{Distribution}

Helicops tapajonicus is known only from two localities at the river Tapajos close to its confluence with the Amazon in the Brazilian state Pará (Fig. 3e). 


\section{Helicops trivittatus (Gray, 1849)}

\section{Materials}

a. $\quad$ scientificName: Helicops trivittatus (Gray, 1849); year: 1915; individualCount: 1; sex: female; catalogNumber: SMF 17798; recordedBy: Z.G. leg.; institutionID: SMF

b. $\quad$ scientificName: Helicops trivittatus (Gray, 1849); country: Brazil; stateProvince: Para; locality: Amazonas; year: 1953; individualCount: 1; sex: male; catalogNumber: SMF 45434; recordedBy: K. Müller leg.; institutionID: SMF

c. $\quad$ scientificName: Helicops trivittatus (Gray, 1849); country: Brazil; stateProvince: Pará; locality: Insel Marajó, Cachoeira am mittleren Arary; year: 1910; individualCount: 1; sex: female; catalogNumber: ZSM 272/2017; recordedBy: L. Müller leg.; institutionID: ZSM

d. $\quad$ scientificName: Helicops trivittatus (Gray, 1849); country: Brazil; stateProvince: Pará; locality: Insel Marajó, Cachoeira am mittleren Arary; year: 1910; individualCount: 1; sex: female; catalogNumber: ZSM 273/2017; recordedBy: L. Müller leg.; institutionID: ZSM

\section{Diagnosis}

This species can be distinguished from all congeners by the combination of having five narrow light stripes on the dorsum and a light venter with black semilunar markings, which extend onto the tail (for information on references see Suppl. material 3).

\section{Distribution}

Helicops trivittatus is present from the eastern part of the Brazilian province Para to approximately its borders with Maranhao and Tocantins and the northern Mato Grosso. There are no reports of this species from western Para (Fig. 3f).

\section{Morphology remark}

The presence of intergenials seems to be a reliable identification character in the remaining species in this genus, whereas in $H$. trivittatus this character shows considerable variation. Intergenials are sometimes present, and there is no geographical pattern perceivable (pers. Com. Antonio Moraes).

\section{Helicops yacu Rossman \& Dixon, 1975}

\section{Diagnosis}

Helicops yacu can be distinguished from all congeners by the combination of having an entire nasal scale, and intergenials present (for information on references see Suppl. material 3).

\section{Distribution}

Helicops yacu is known only from the Isla Yanamono in the Peruvian province Loreto (Rossman and Dixon 1975, Fig. 4). 


\section{Taxonomic remark}

In Rossman and Abe (1979) the authors express their doubt that $H$. yacu represents an own species. In this publication the authors suggest that it might represent a subspecies of $H$. pastazae. No further examination of this suggestion has been made since then.

\section{Helicops sp.}

\section{Material}

a. scientificName: Helicops sp.; country: Brazil; stateProvince: Pernambuco; year: 1913-1914; individualCount: 1; sex: female; catalogNumber: SMF 34035; recordedBy: E. Bresslau leg.; institutionID: SMF

\section{Diagnosis}

The female specimen SMF 34035 is distinguished from all other congeners except $H$. infrataeniatus by having 17 dorsal scale rows at midbody and posterior body and 124 ventrals. It differs from $H$. infrataeniatus in having a black venter with cream, narrow transversal bands, which are approximately a ventral scale wide, often left and right halves are shifted one ventral scale, forming a pattern resembling a chessboard. Helicops infrataeniatus has a venter either with three black stripes on a cream background or checkered black and cream, sometimes red. Additionally, the specimen is separated from $H$. infrataeniatus by its distribution. It originates from the Brazilian province Pernambuco, whereas $H$. infrataeniatus is occurring not further north than Mato Grosso do Sul in Brazil (Pholidosis of speciemen 34035 see Table 2; for information on references see Suppl. material 3).

\section{Distribution}

The specimen originates from the province Pernambuco in Brazil, no exact locality given.

\section{Identification keys}

\section{Identification key to the species of Helicops Wagler, 1830}

Dichotomous identification key created based on own examinations and literature listed in Suppl. material 3.

1 Dorsum uniform or with longitudinal stripes

- Dorsum with blotches, spots, or transverse bars 
2

Dorsum tan to dark brown with five rows of narrow light stripes, ventral cream with 2 uniform rows of dark brown to black semilunar marks

Helicops trivittatus

- Coloration not as above

3

3 Venter cream or yellow with 2-3 rows of black semilunar marks, 9-10 infralabials, $128-141$ ventrals in males and 128-148 ventrals in females

H. carinicaudus

Coloration and pholidosis not as above

- Coloration and pholidosis not as above

\begin{tabular}{l|l|l}
4 & Nasal entire & 7
\end{tabular}

- Nasal semidivided $\quad 5$

5 Ventral body cream, with or without faint brown flecks $\quad$ H. modestus

- Venter contrastingly checkered or with dark longitudinal stripes $\quad 6$

6 Dorsum uniform moss green, dorsal weakly keeled

H. tapajonicus

Venter cream or red with 1-3 dark stripes or darkly checkered or black

- $\quad$ with light spots or intermediate forms; dorsal strongly keeled; dorsum

H. infrataeniatus dark brown with pale brown stripes

Dorsal scale rows at midbody 17 , reducing to 15 anterior to cloaca; 56

7 subcaudals in single known male, 41-52 in females; dorsum uniform dark olive, dark brown, or dark gray

H. nentur

Dorsal scale rows at midbody 25 , reducing to 21 anterior to cloaca; 68 subcaudals in single known male, unknown in females; dorsum greenish copper brown with three longitudinal chainlike rows of dark spots, Venter

H. boitata light greyish brown with two lateral rows of light orange spots

8 Intergenials present

9

- Intergenials absent

Nasal entire; 85-96 subcaudals in females, unknown in males; $25-28$

9 dorsal scale rows at midbody, reducing to $18-20$ anterior to cloaca; dorsum light to medium gray brown with 4 alternating rows of relatively H. yacu small dark spots

Nasal semidivided; 55-117 subcaudals in males, 51-97 in females; 19

- 29 dorsal scale rows at midbody, reducing to 16-23 anterior to cloaca; coloration variable

10

55-67 subcaudals in males, $50-53$ in females; dorsum gray brown with alternating light and dark circular blotches; northern South America

H. hagmanni 
- $\quad$ 83-117 subcaudals in males, 50-97 in females; coloration variable

110-119 ventrals in males, 113-125 in females; subcaudal keels absent;

11 dorsum grayish tan with 3-5 rows of irregular dark blotches, the vertebral blotches larger than laterals, all 3 usually fused longitudinally; northern South America

121-142 subcaudals in males, $130-150$ in females; subcaudal keels present; coloration variable

Weak subcaudal keels present, $121-134$ ventrals in males, $130-145$ in females; $93-117$ subcaudals in males, $72-97$ in females; $23-25$ dorsal scale rows at midbody, reducing to 16-19 anterior to cloaca; ventral coloration cream with a series of dark crossbands or alternating checks, light ventral color extending onto several dorsal scale rows; norther South America

Subcaudal keels absent, 135-142 ventrals in males, 137-150 in females; 85-91 subcaudals in males, $67-73$ in females; $21-23$ dorsal scale rows at midbody, reducing to 16 anterior to cloaca; ventral coloration cream with a lateral series of dark checks; eastern Andean foothills of Ecuador

13 Subcaudal keels present

- Subcaudal keels absent

103-123 ventrals in males, $104-125$ in females; $17-20$ dorsal scale rows at midbody

118-132 ventrals in males, 124-132 in females, or if fewer than 124 ventrals in males, then $21-22$ dorsal scale rows at midbody

19 dorsal scale rows at midbody; dorsum with dark blotches; one anterior

15 temporal; 71-86 subcaudals in males, 67-73 in females; 125-132 ventrals in males, 128-132 in females

21-24 dorsal scale rows at midbody; dorsum with dark transverse bands; 2-3 anterior temporals; 79-103 subcaudals in males, 80-84 in females; 118-127 ventrals in males, $124-132$ in females; northern Mato Grosso, Brazil

Dorsum scale rows at midbody $23-26$, reducing to $17-21$ anterior to cloaca; $71-101$ subcaudals in males, $71-88$ in females; $10-13$ infralabials; venter dark with pale spots
H. scalaris

12

H. pastazae

H. petersi

14

16

$H$. angulatus

15

H. gomesi

H. apiaka

H. polylepis 


\begin{tabular}{|l|l|l|}
\hline $\begin{array}{l}\text { Dorsum scale rows at midbody 19-22, reducing to 16-19 anterior to } \\
\text { cloaca; } 64-89 \text { subcaudals in males, 53-76 in females; 8-11 infralabials; } \\
\text { venter checkered or banded black and red, or cream with two medial } \\
\text { rows of black semilunar marks, sometimes fused midventrally }\end{array}$ & 17 \\
\hline 17 & $\begin{array}{l}19 \text { dorsal scale rows at anterior and midbody and 17-19 dorsal scale } \\
\text { rows anterior to cloaca; dorsal scales with moderate keels; dark dorsal } \\
\text { spots fusing to transversal bands }\end{array}$ & H. phantasma \\
\hline- & $\begin{array}{l}\text { Number of dorsal scale rows different } \\
\text { Venter checkered or banded black and red; 108-129 ventrals in males, } \\
108-138 \text { in females }\end{array}$ & H. leopardinus \\
\hline Venter cream with two medial rows of black semilunar marks, sometimes & H. danieli \\
\hline fused midventrally; 125-135 ventrals in males, 130-141 in females & \\
\hline
\end{tabular}

\section{Discussion}

\section{Taxonomic discussion}

We were unable to find a pholidosis character for distinguishing the species $H$. carinicaudus and $H$. infrataeniatus, but found them to differ in the coloration of the venter. Helicops carinicaudus has a yellow or cream venter with two series of black semilunar marks with small, irregular black spots between these marks, versus venter red to white, with three black stripes, checkered black and light, or black with light spots in $H$. infrataeniatus. Obviously, these species need a closer examination using molecular methods. A similar assessment for $H$. infrataeniatus was made by Achaval Elena (2001), who evaluated the systematics and distribution of most reptile species in Uruguay. A further hint for the necessity of revisionary work on these species is the documentation of Helicops sp. reported in this publication. As described, it resembles $H$. infrataeniatus, but has a considerably different ventral coloration.

\section{Geographical distribution}

When comparing the examined specimens to the literature used to create the point distribution maps we can report range extensions for six species. For $\mathrm{H}$. angulatus we here report the first records in French Guyana (ZMB 2305 and ZMB 54167), the province Chapare in Bolivia (ZSM 516/2003) and the province Maranhao in Brazil (ZMB 10854). All four specimens did not possess a precise locality description. For Helicops danieli we report the first specimen originating from Brazil. The specimen ZMB 9490 has no preciser locality description. The nearest literature report of this species to Brazil is only $20 \mathrm{~km}$ away from the Columbian-Brazilian border at Mitu, in the Columbian province Vaupes (Yuki and Castano 1998). We report two range extensions for $H$. infrataeniatus. We report the extension of the distribution range more than $600 \mathrm{~km}$ to the South from the nearest 
literature report by Entiauspe-Neto et al. (2016) with the specimens ZFMK 102499 and ZFMK 30350, which were collected at Punta Lara, Buenos Aires, Argentina. The specimen SMF 67327 extends the distribution range of this species over $400 \mathrm{~km}$ further north from the nearest report by Arzamendia and Giraudo (2002). It was collected in Roque Saenz Pena, Chaco, Argentina. For the species $H$. leopardinus we report the extension of the distribution range to the departamento Beni in Bolivia with the speciemens ZFMK 62836, collected at Campamento Encanto, Beni, Bolivia, and ZSM 134/1947, collected at the Rio Madre de Dios, Beni, Bolivia. The specimen MTKD 27443 was found near San Francisco, Cordoba, Argentina. This specimen represents the first record of this species in this province, extending the distribution range around $150 \mathrm{~km}$ from the nearest encounter report by Taub (1967). We extend the distribution range of Helicops pastazae to the province Napo, Ecuador with the specimen ZMB 519/2003. It was collected in Virgilio Davila, Quijos, Napo, Ecuador. This is around $90 \mathrm{~km}$ east from the nearest literature report at Yachana Reserve, Orellana, Ecuador by Whitworth and Beirne (2011). It is conspicuous, that there are specimens from Eastern Venezuela and from northern Ecuador, but no specimens or reports from Columbia. It is likely, that $H$. pastazae is present in Columbia, but has not been reported, due to missing research and fieldwork. For the species $H$. polylepis we report the first record from the province La Paz in Bolivia. The specimen SMF 17821 was collected in La Paz, La Paz, Bolivia. This extends the known distribution of the species about $400 \mathrm{~km}$ to the south from eastern Madre de Dios, Peru (Flores et al. 2010). Furthermore, we report the first specimen from the province Chuquisaca in Bolivia. The specimen ZMB 26215 was collected in Chaco, Chuquisaca, Bolivia. This extends the known distribution range over $800 \mathrm{~km}$ to the southwest from the nearest record by Griffin (1916) in southern Mato Grosso. The last range extension we report for this species is to the province Bahia in Brazil. The specimen ZMB 17428 was collected there, but no exact locality description is available.

The number of range extensions we report here is a sign, that the distribution range of the species in this genus is not well known. To get a better knowledge about the distribution a comprehensive examination of collection material on an international level and especially field work in the aquatic habitat of this genus is required.

\section{Acknowledgements}

We particularly want to thank Linda Mogk, Martin Jansen, Marcel Nebenführ and Joseph Vargas (all SMF), who always provided a honest opinion and friendly support. Furthermore, we thank Antonio Moraes da Silva, Rafaela França, Ricardo Alexandre Kawashita-Ribeiro, Omar Entiauspe, Santiago Carreira and Pier Cacciali for corresponding with us and sharing their knowledge with us. Futhermore we thank the curators/collection managers permitting us access to their collections and providing additional information: Raffael Ernst (MTKD), Markus Auer (MTKD), Alexander Kupfer (SMNS), Mark Oliver Rödel (ZMB), Frank Tillack (ZMB), Claudia Koch (ZFMK), Morris Flecks (ZFMK), Frank Glaw (ZSM), Michael Franzen (ZSM). 


\section{Author contributions}

YS did the literature research, examination of the specimens and the writing of the publication. GK was the academic supervisor and revised the manuscript.

\section{References}

- Achaval Elena F (2001) Systematic update and maps of distribution of the reptiles of Uruguay. Actualizacion sistematica y mapas de distribucion de los reptiles del Uruguay. Smithsonian Herpetological Information Service 129.

- $\quad$ Arzamendia V, Giraudo AR (2002) Lista y distribución de los ofidios (Reptilia: Serpentes) de Santa Fe, Argentina. Cuadernos de Herpetología 16 (1). URL: http:// sedici.unlp.edu.ar/bitstream/handle/10915/6363/Documento completo.pdf?sequence=1

- $\quad$ Carreira Vidal S, Meneghel M, Achaval F (2005) Reptiles de Uruguay. Universidad de la República, Facultad de Ciencias, Montevideo.

- $\quad$ Costa H, Santana D, Leal F, Koroiva R, Garcia PA (2016) A new species of Helicops (Serpentes: Dipsadidae: Hydropsini) from southeastern Brazil. Herpetologica 72 (2): 157-166. https://doi.org/10.1655/HERPETOLOGICA-D-15-00059

- Di Pietro DO, Alcalde L, Williams JD (2014) Nasal cartilages, hyobranchial apparatus, larynx, and glottal tubes in four species of Hydropsini (Serpentes: Dipsadidae: Xenodontinae). Vertebrate Zoology 64 (1): 103-111.

- Dowling H (1951) A proposed standard system of counting ventrals in snakes. British Journal of Herpetology 1: 97-99.

- $\quad$ Entiauspe-Neto OM, Perleberg TD, de Freitas MA (2016) Herpetofauna from an urban Pampa fragment in southern Brazil: composition, structure and conservation. Check List 12 (5). https://doi.org/10.15560/12.5.1964

- $\quad$ Flores E, Jadin R, Orlofske S (2010) Helicops polylepis (Norman's Keelback). Diet and intraspecific competition. Herpetological Review 41 (1): 93-94.

- França RCd, Germano CEdS, França FGR (2012) Composição de uma taxocenose de serpentes em uma área urbana na Mata Atlântica da Paraíba, Nordeste do Brasil. Copeia 12 (3): 183-195. https://doi.org/10.1590/S1676-06032012000300019

- Griffin LE (1916) A catalog of the Ophidia from South America at present (June, 1916) contained in the Carnegie Museum, with descriptions of some new species. Carnegie Institute, Pittsburgh.

- Hernández-Ruiz EJ, Wariss Figueiredo M, Brito Pezzuti JC (2014) Bycatch of Helicops angulatus (Linnaeus, 1758) (Reptilia: Squamata: Colubridae) in hoop-traps used to capture fresh water turtles on the coast fo Pará, Brazil. Acta Biológica Colombiana 19 (1): 119-120.

- $\quad$ Kawashita-Ribeiro RA, Ávila RW, Morais DH (2013) A new snake of the Genus Helicops Wagler, 1830 (Dipsadidae, Xenodontinae) from Brazil. Herpetologica 69 (1): 80-90. https://doi.org/10.1655/HERPETOLOGICA-D-12-00013

- $\quad$ Koski DA, Monico AT, Koski AP (2016) Helicops angulatus (Brown-banded Watersnake). Predation. Herpetological Review 47 (3): 478-479. 
- Linnaeus C (1758) Systema naturae (Systema naturae per regna tria naturae, secundum classes, ordines, genera, species, cum characteribus, differentiis, synonymis, locis. Tomus I. Editio decima, reformata). 10, 1. Lars Salvi, Stockholm.

- Moraes-da-Silva A, Amaro RC, Nunes Sales P, Strüssmann C, Teixeira Junior M, Andrade A, Sudre V, Recoder R, Rodrigues Trefaut M, Curcio FF (2019) Chance, luck and a fortunate finding: a new species of watersnake of the genus Helicops Wagler, 1828 (Serpentes: Xenodontinae), from the Brazilian Pantanal wetlands. Zootaxa 4651 (3): 445-470. https://doi.org/10.11646/zootaxa.4651.3.3

- Moraes-da-Silva A, Amaro RC, Nunes PS, Rodrigues MT, Curcio FF (2021) Long known, brand new, and possibly threatened: a new species of watersnake of the genus Helicops Wagler, 1828 (Serpentes; Xenodontinae) from the Tocantins-Araguaia River Basin, Brazil. Zootaxa 4903 (2): 217-241. https://doi.org/10.11646/zootaxa.4903.2.3

- Peters JA, Orejas-Miranda B (1970) Catalogue of the Neotropical Squamata: Part I. Snakes. United States National Museum Bulletin 297.

- $\quad$ Rossman DA, Dixon JR (1975) A new colubrid snake of the genus Helicops from Peru. Herpetologica 31 (4): 412-414.

- Rossman DA, Abe AS (1979) Comments on the taxonomic status of Helicops yacu (Serpentes: Colubridae). Proceedings of the Louisiana Academy of Sciences 42 (7-9).

- Rossman DA (2002) Variation in the xenodontid water snake Helicops scalaris Jan, and the status of $H$. hogei Lancini. Occasional Papers of the Museum of Natural Science 78: 1-18.

- $\quad$ Silva Jr NJ (1993) The snakes from Samuel hydroelectric power plant and vicinity, Rondônia, Brazil. Herpetological Natural History 1 (1): 37-86.

- Taub A (1967) Comparative histological studies on Duvernoy's gland of colubrid snakes. Bulletin of the American Museum of Natural History 138.

- Vaz-Silva W, Oliveira R, Gonzaga A, Pinto K, Poli F, Bilce T, Penhacek M, Wronski L, Martins J, Junqueira T, Cesca L, Guimarães V, Pinheiro R (2015) Contribuições para o conhecimento de anfíbios e répteis da Volta Grande do Xingu, norte do Brasil. Brazilian Journal of Biology 75 (3): 205-218. https://doi.org/10.1590/1519-6984.00814BM

- Whitworth A, Beirne C (2011) Reptiles of the Yachana Reserve. Global Vision International, Exeter. https://doi.org/10.13140/RG.2.1.4130.6968

- Yuki RN, Castano OV (1998) Geographic distribution note of water-snake Helicops danieli Amaral, 1937 (Colubridae: Xenodontinae). The Snake 28 (1-2): 90-92. 


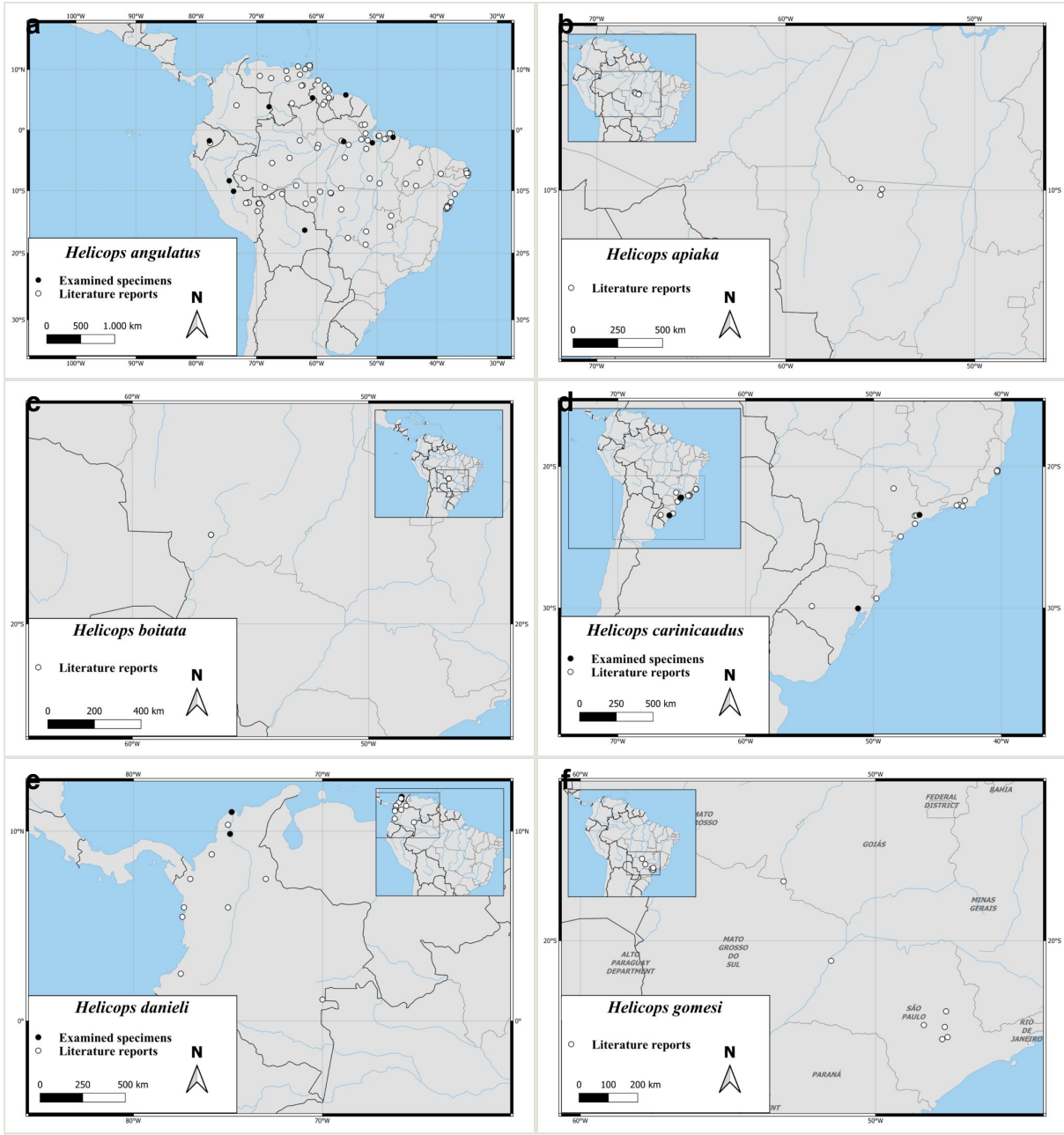

Figure 1.

Distribution maps of A Helicops angulatus B $H$. apiaka $\mathbf{C}$. boitata D $H$. carinicaudus E $H$. danieli $\mathbf{F} \mathbf{H}$. gomesi. Black circles indicate examined specimens and white circles literature reports. For the coordinates and references of the distribution points from literature see Suppl. material 2. 


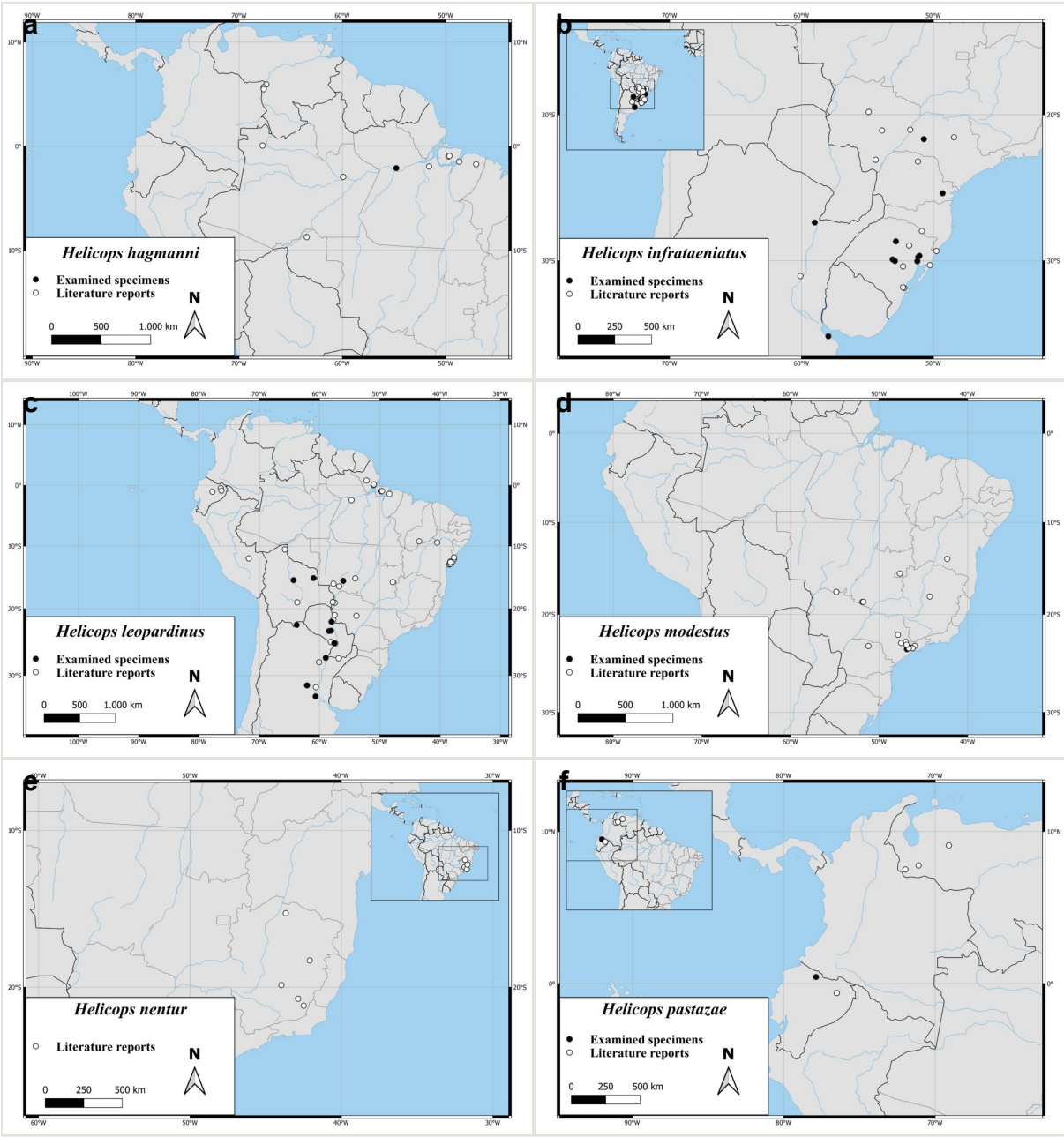

Figure 2.

Distribution maps of A Helicops hagmanni B H. infrataeniatus C H. leopardinus $\mathbf{D}$ H. modestus E $H$. nentur $\mathbf{F}$. pastazae. Black circles indicate examined specimens and white circles literature reports. For the coordinates and references of the distribution points from literature see Suppl. material 2. 

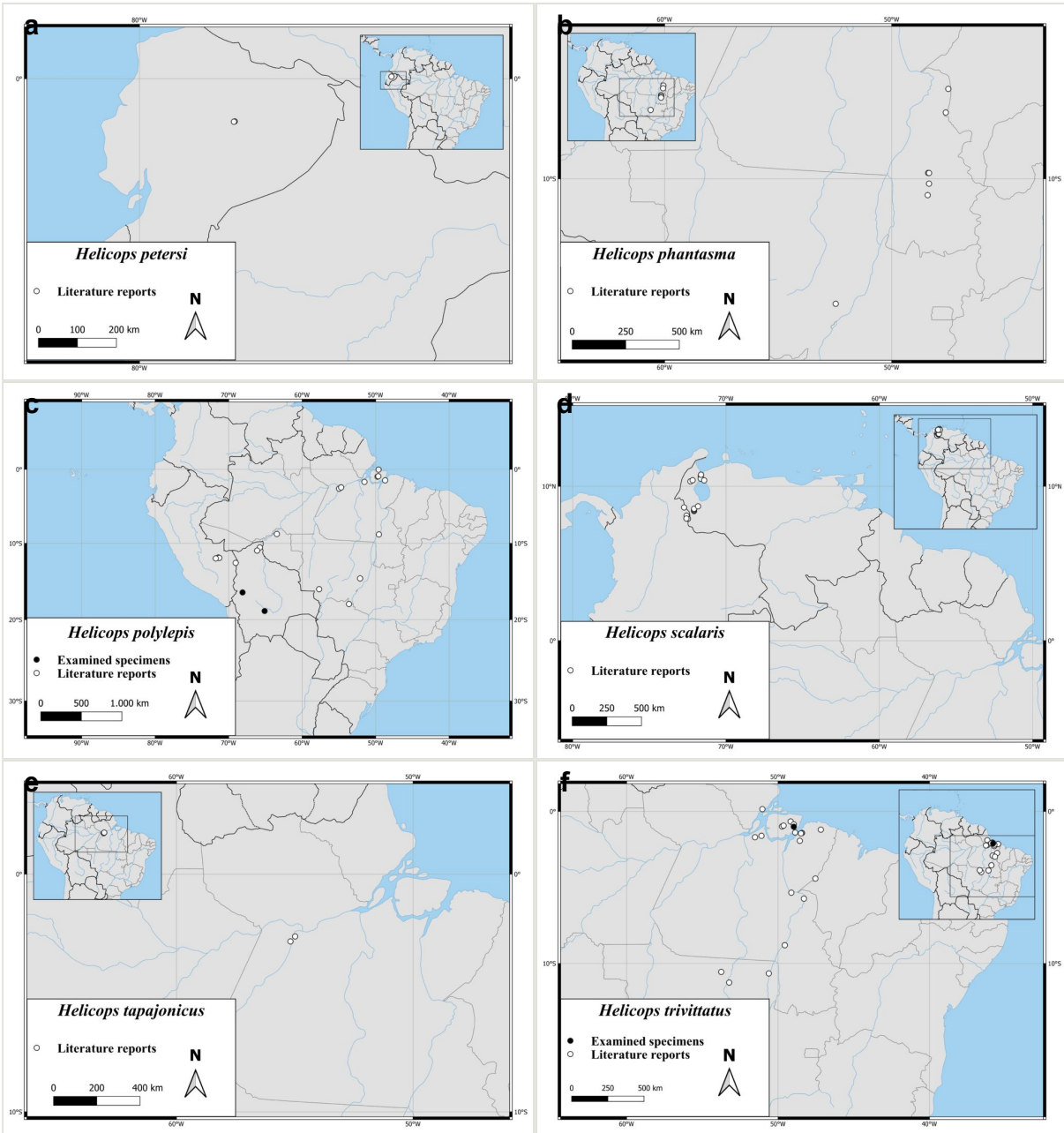

Figure 3.

Distribution maps of A Helicops petersi B $H$. phantasma $\mathbf{C} H$. polylepis $\mathbf{D} H$. scalaris $\mathbf{E} H$. tapajonicus $\mathbf{F} \boldsymbol{H}$. trivittatus. Black circles indicate examined specimens and white circles literature reports. For the coordinates and references of the distribution points from literature see Suppl. material 2. 


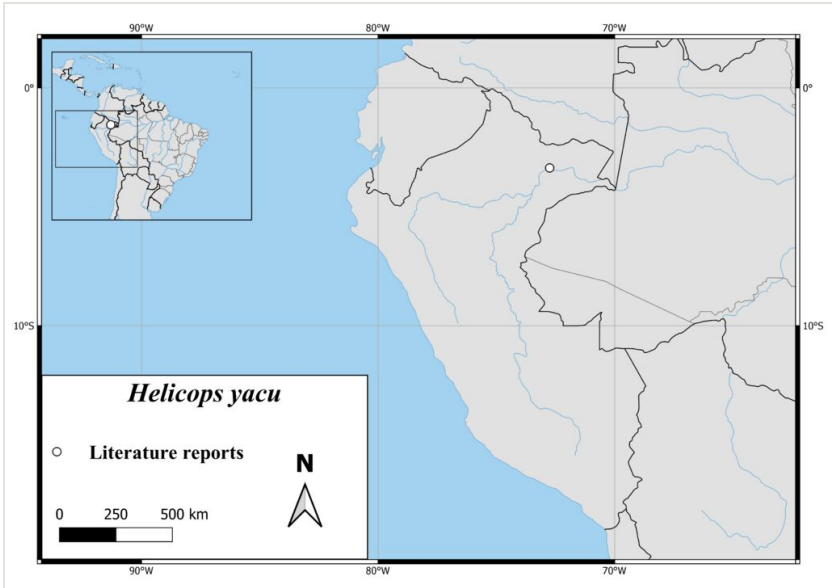

Figure 4.

Distribution of Helicops yacu. White circles represent literature reports. For the coordinates and references of the distribution points from literature see Suppl. material 2. 
Table 1.

Summarized results of the morphologic examination of 190 specimens. Abbreviations: SVL: snoutvent length; TL: tail length; VE: ventrals; SC: subcaudals; SCK: presence of subcaudal keels; LO: loreals; PRO: preoculars; PSO: postoculars; AT: anterior temporals; PT: posterior temporals; SL: supralabials; SL+E: supralabials in contact with the eye; IL: infralabials; DSM: dorsal scale rows at midbody; DKM: dorsal keels at midbody; DSP: dorsal scale rows at posterior body; DKP: dorsal keels at posterior body; CL: cloacal plate; div: divided; IG: intergenials; NA: if nasal is divided; sdiv: semidivided.; values in brackets indicate observations we rate as natural abnormalities, which are discussed in the respective species account. Values were rounded to the third decimal place, lengths are in milimeters. See Suppl. material 1 for data of all specimens

\begin{tabular}{|c|c|c|c|c|c|c|c|}
\hline & H. angulatus & $\begin{array}{l}\text { H. } \\
\text { carinicaudus }\end{array}$ & $\begin{array}{l}H . \\
\text { danieli }\end{array}$ & H. hagmanni & $\begin{array}{l}\mathrm{H} . \\
\text { infrataeniatus }\end{array}$ & H. leopardinus & H. modestus \\
\hline $\begin{array}{l}\text { SVL } \\
\hat{0}\end{array}$ & $\begin{array}{c}229 \\
- \\
420\end{array}$ & $\begin{array}{c}414 \\
- \\
570\end{array}$ & 409 & 460 & $\begin{array}{c}157 \\
- \\
489\end{array}$ & $\begin{array}{c}217 \\
- \\
495\end{array}$ & $\begin{array}{c}165 \\
- \\
305\end{array}$ \\
\hline $\begin{array}{l}\text { SVL } \\
q\end{array}$ & $\begin{array}{c}145 \\
- \\
680\end{array}$ & $\begin{array}{c}280 \\
- \\
810\end{array}$ & $\begin{array}{c}163 \\
- \\
620\end{array}$ & 575 & $\begin{array}{c}136 \\
- \\
600\end{array}$ & $\begin{array}{c}139 \\
- \\
620\end{array}$ & $\begin{array}{c}98 \\
- \\
438\end{array}$ \\
\hline $\begin{array}{l}\text { TL } \\
\hat{\delta}\end{array}$ & $\begin{array}{c}128 \\
- \\
275\end{array}$ & $\begin{array}{c}160 \\
- \\
190\end{array}$ & 194 & 174 & $\begin{array}{c}66 \\
- \\
174\end{array}$ & $\begin{array}{c}114 \\
- \\
194\end{array}$ & $\begin{array}{c}68 \\
- \\
125\end{array}$ \\
\hline $\begin{array}{l}\text { TL } \\
\text { q }\end{array}$ & $\begin{array}{c}30 \\
- \\
325\end{array}$ & $\begin{array}{c}90 \\
- \\
203\end{array}$ & $\begin{array}{c}62 \\
- \\
185\end{array}$ & 185 & $\begin{array}{c}45 \\
- \\
194\end{array}$ & $\begin{array}{c}53 \\
- \\
235\end{array}$ & $\begin{array}{c}33 \\
- \\
129\end{array}$ \\
\hline $\begin{array}{l}\text { TL/ } \\
\text { SVL } \\
\delta\end{array}$ & $\begin{array}{c}0.417 \\
- \\
0.696\end{array}$ & $\begin{array}{c}0.330 \\
- \\
0.387\end{array}$ & 0.474 & 0.378 & $\begin{array}{c}0.311 \\
- \\
0.526\end{array}$ & $\begin{array}{c}0.333 \\
- \\
0.547\end{array}$ & $\begin{array}{c}0.410 \\
- \\
0.412\end{array}$ \\
\hline $\begin{array}{l}\text { TL/ } \\
\text { SVL } \\
q\end{array}$ & $\begin{array}{c}0.185 \\
- \\
0.922\end{array}$ & $\begin{array}{c}0.235 \\
- \\
0.321\end{array}$ & $\begin{array}{c}0.271 \\
- \\
0.380\end{array}$ & 0.323 & $\begin{array}{c}0.209 \\
- \\
0.462\end{array}$ & $\begin{array}{c}0.313 \\
- \\
0.557\end{array}$ & $\begin{array}{c}0.295 \\
- \\
0.432\end{array}$ \\
\hline $\begin{array}{l}\text { VE } \\
\delta\end{array}$ & $\begin{array}{c}103 \\
- \\
119\end{array}$ & $\begin{array}{c}139 \\
- \\
141\end{array}$ & 128 & 123 & $\begin{array}{c}114 \\
- \\
128\end{array}$ & $\begin{array}{c}110 \\
- \\
129\end{array}$ & $\begin{array}{c}114 \\
- \\
116\end{array}$ \\
\hline $\begin{array}{l}\text { VE } \\
\text { q }\end{array}$ & $\begin{array}{c}104 \\
- \\
125 \\
(130 ; 156)\end{array}$ & $\begin{array}{c}128 \\
- \\
146\end{array}$ & $\begin{array}{c}131 \\
- \\
139\end{array}$ & 131 & $\begin{array}{c}113 \\
- \\
130\end{array}$ & $\begin{array}{c}109 \\
- \\
127\end{array}$ & $\begin{array}{c}112 \\
- \\
124\end{array}$ \\
\hline $\begin{array}{l}\text { SC } \\
\delta\end{array}$ & $\begin{array}{c}69 \\
- \\
100\end{array}$ & $\begin{array}{c}64 \\
- \\
71\end{array}$ & 79 & 59 & $\begin{array}{l}58 \\
- \\
88\end{array}$ & $\begin{array}{l}53 \\
- \\
88\end{array}$ & $\begin{array}{c}64 \\
- \\
67\end{array}$ \\
\hline $\begin{array}{l}\text { SC } \\
+\end{array}$ & $\begin{array}{c}58 \\
- \\
92\end{array}$ & $\begin{array}{l}51 \\
- \\
59\end{array}$ & $\begin{array}{l}60 \\
- \\
81\end{array}$ & 50 & $\begin{array}{c}49 \\
- \\
74\end{array}$ & $\begin{array}{c}56 \\
- \\
88 \\
(109)\end{array}$ & $\begin{array}{c}49 \\
- \\
70\end{array}$ \\
\hline SCK & present & absent & absent & present & $\begin{array}{c}\text { absent } \\
\text { (1x present) }\end{array}$ & absent & absent \\
\hline
\end{tabular}




\begin{tabular}{|c|c|c|c|c|c|c|c|}
\hline LO & 1 & $1-2$ & 1 & 1 & $0-3$ & $0-2$ & $0-2$ \\
\hline PRO & $1-2$ & $1-2$ & 1 & 1 & $1-2$ & $1-2$ & $1-2$ \\
\hline PSO & $2-3$ & 2 & 2 & $1-2$ & 2 & $1-2$ & 2 \\
\hline AT & $1-3$ & 1 & 1 & 1 & $1-2$ & $1-2$ & 1 \\
\hline PT & $2-4$ & $1-2$ & $2-3$ & 3 & $1-3$ & $1-3$ & 2 \\
\hline SL & $8-9$ & $7-8$ & $7-8$ & 8 & $7-8$ & $7-9$ & $7-8$ \\
\hline $\mathrm{SL}+\mathrm{E}$ & IV & IV, III-IV & $\begin{array}{l}\text { IV, } \\
\text { IV-V }\end{array}$ & IV & $\begin{array}{l}\text { III, III-IV, } \\
\text { IV }\end{array}$ & III-IV, IV, IV-V & III-IV, IV \\
\hline IL & $9-11$ & $9-10$ & $9-12$ & $11-12$ & $9-12$ & $9-11$ & $9-11$ \\
\hline DSM & $17-20$ & $17-19$ & $18-19$ & $25-27$ & $17-20$ & $18-19$ & $17-20$ \\
\hline DSM & present & present & present & present & present & present & present \\
\hline DRP & $16-17$ & 17 & 17 & 21 & $15-17$ & $16-19$ & $15-19$ \\
\hline DKP & present & present & present & present & present & present & present \\
\hline $\mathrm{CL}$ & div & div & div & div & div & div & div \\
\hline IG & absent & absent & absent & present & absent & absent & absent \\
\hline NA & sdiv & sdiv & sdiv & sdiv & sdiv & sdiv & sdiv \\
\hline
\end{tabular}


Table 2.

Pholidosis characters of the female specimen SMF 34035. Abbreviations: SVL: snout-vent length; TL: tail length; VE: ventrals; SC: subcaudals; presence of subcaudal keels (SCK); PRO: preoculars; PSO: postoculars; LO: loreal; AT: anterior temporals; NA: nasal; PT: posterior temporals; SL: supralabials; SL+E: supralabials in contact with the eye; IL: infralabials; DSM: dorsal scale rows at midbody; DKM: dorsal keels at midbody; DSP: dorsal scale rows at posterior body; DKP: dorsal keels at posterior body; CL: cloacal plate; IG: presence of Intergenials; Decimal values were rounded to the third decimal place, lengths are in milimeters. See Suppl. material 1 for data of all specimens.

\begin{tabular}{|l|l|l|l|}
\hline SVL & 365 & PT right & 2 \\
\hline TL & 189 & PT left & 2 \\
\hline TL/SVL & 0,518 & SL right & 8 \\
\hline VE & 124 & SL left & 8 \\
\hline SC & 75 & SL+E right & IV \\
\hline SCK & absent & SL+E left & IV \\
\hline PRO right & 1 & IL right & 10 \\
\hline PRO left & 1 & IL left & 10 \\
\hline LO right & 1 & DSM & 17 \\
\hline LO left & 1 & DKM & present \\
\hline PSO right & 2 & DSP & 17 \\
\hline PSO left & 2 & DKP & present \\
\hline AT right & 1 & CL & divided \\
\hline AT left & 1 & IG & absent \\
\hline NA & semidivided & & \\
\hline & & & \\
\hline
\end{tabular}




\section{Supplementary materials}

\section{Suppl. material 1: Examination results}

Authors: Yannis Schöneberg, Gunther Köhler Data type: morphological

Brief description: Examination results of all 190 specimens examined in this study.

Download file $(26.03 \mathrm{~kb})$

\section{Suppl. material 2: References for all locality records extracted from literature}

Authors: Yannis Schöneberg, Gunther Köhler

Data type: occurences

Brief description: This table contains the description, coordinates and the reference of all used distribution points, which were extracted from literature.

Download file (112.61 kb)

\section{Suppl. material 3: References for the morphological data}

Authors: Yannis Schöneberg, Gunther Köhler

Data type: morphological

Brief description: This file contains all references used for the assessment of the morphological traits.

Download file $(11.98 \mathrm{~kb})$ 